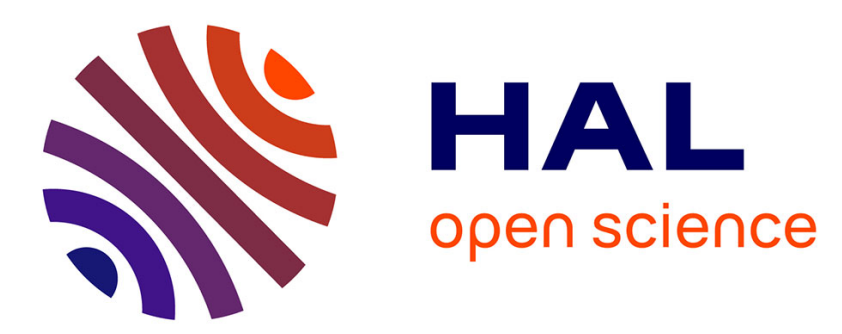

\title{
Le mobilier céramique de l'Âge du Bronze final III des sites des Estournelles et de la Plaine à Simandres
} (Rhône)

Franck Thiériot

\section{- To cite this version:}

Franck Thiériot. Le mobilier céramique de l'Âge du Bronze final III des sites des Estournelles et de la Plaine à Simandres (Rhône). Bulletin de la Société préhistorique française, 2005. hal-02365221

\section{HAL Id: hal-02365221 https://hal-inrap.archives-ouvertes.fr/hal-02365221}

Submitted on 19 Nov 2019

HAL is a multi-disciplinary open access archive for the deposit and dissemination of scientific research documents, whether they are published or not. The documents may come from teaching and research institutions in France or abroad, or from public or private research centers.
L'archive ouverte pluridisciplinaire HAL, est destinée au dépôt et à la diffusion de documents scientifiques de niveau recherche, publiés ou non, émanant des établissements d'enseignement et de recherche français ou étrangers, des laboratoires publics ou privés. 


\title{
Le mobilier céramique de l'Âge du Bronze final III des sites des Estournelles et de la Plaine à Simandres (Rhône)
}

\begin{abstract}
Résumé
L'examen du mobilier céramique des sites des Estournelles et de la Plaine à Simandres et sa confrontation à celui d'autres séries du nord de la région Rhône-Alpes permettent de déterminer un certain nombre de caractéristiques typologiques propres au $X^{e}$ siècle av. J.-C. (Bronze final IIIa). Quelques hypothèses chronologiques et géographiques concernant l'impact et l'évolution des influences du groupe Rhin-Suisse-France orientale dans un secteur concernant la moyenne vallée du Rhône français sont ensuite proposées.
\end{abstract}

\begin{abstract}
Examination of the ceramics from the sites of Les Estournelles and La Plaine at Simandres and their comparison with other series from the north of the Rhone-Alpes region allow a certain number of typological characteristics specific to the $10^{\text {th }}$ century $B C$ (Late Bronze $3 a$ ) to be determined. Some chronological and geographical hypotheses concerning the impact and evolution of the influences of the Rhine-Switzerland-Eastern France group in a sector concerning the Middle Rhône Valley in France are then proposed.
\end{abstract}

\section{PRÉSENTATION DES SITES}

Les sites des Estournelles et de la Plaine, dont la fouille fut conduite en 1991 lors des travaux d'aménagement du contournement est de Lyon (A46 Sud), sont localisés à une quinzaine de kilomètres au sud de Lyon (fig. 1). Ils ont livré, entre autres témoins, une série de structures excavées datées de l'Âge du Bronze final dont quatre fosses sépulcrales qui ont permis d'identifier un fonctionnement complexe et original : dépôts primaires d'inhumations, successifs ou simultanés, au sein de fosses circulaires, prélèvement et réenfouissement d'ossements... La présentation des données propres à ces aménagements et à l'ensemble des deux gisements ayant déjà fait l'objet de deux publications
(Thiériot et Saintot, 1999; Blaizot et Thiériot, 2000), nous proposons de renvoyer le lecteur à ces précédentes études pour tous renseignements concernant ces divers aspects. Le présent travail s'intéressera plus particulièrement à l'étude du mobilier céramique de la fin de l'Âge du Bronze, qui, pour des raisons éditoriales, n'avait pu être jointe à ces articles.

\section{DATATIONS ISOTOPIQUES}

Plusieurs structures de ces deux sites ont fait l'objet de datation ${ }^{14} \mathrm{C}$. Les quatre dates ont été effectuées sur charbons de bois de petites dimensions au Centre de datation par le radiocarbone de l'université Claude Bernard-Lyon 1. Les résultats sont donnés en âge 

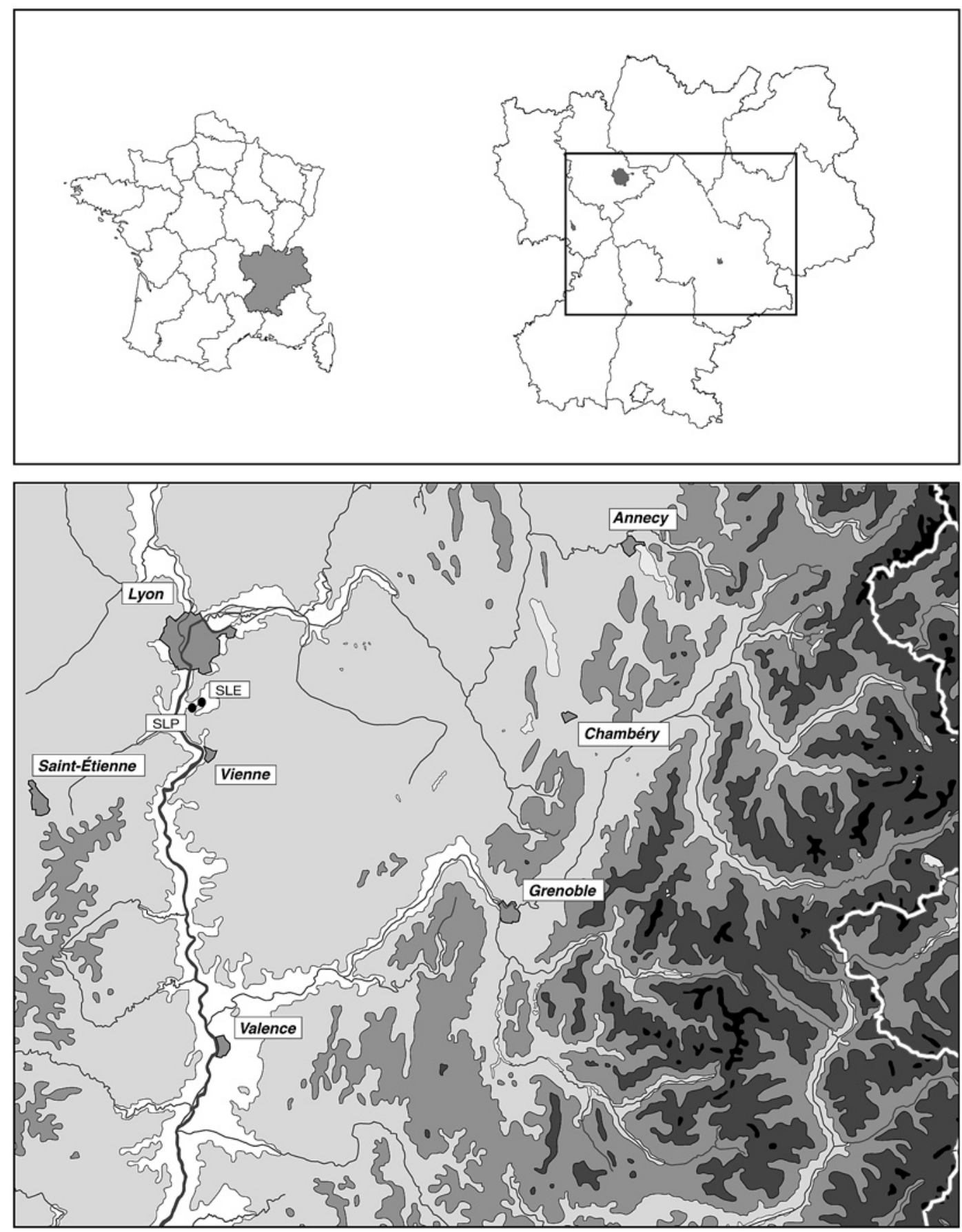

Fig. 1 - Localisation des sites des Estournelles (SLE) et de la Plaine (SLP) à Simandres.

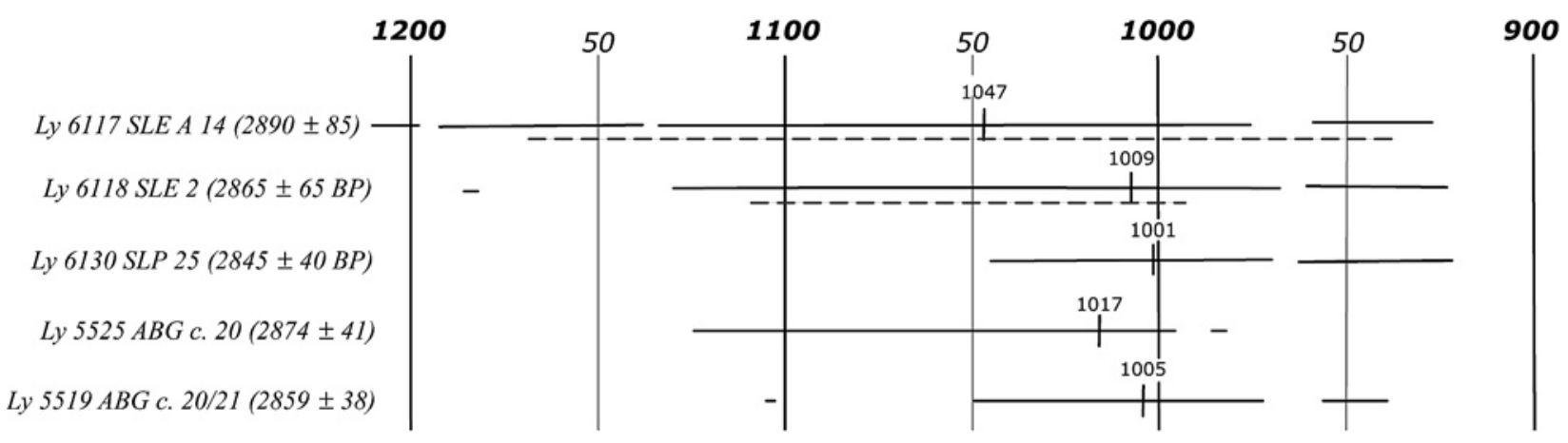

Fig. 2 - Dates ${ }^{14}$ C calibrées des Estournelles, de la Plaine et des couches 20 et 21 de la grotte du Gardon à Ambérieu-en-Bugey (ABG). 
conventionnel BP, puis en âge calibré (Stuiver et Reimer, 1993; Stuiver et al., 1998), selon la méthode des intercepts, avec les points moyens et les intervalles simplifiés à un puis deux sigma (soit $68,3 \%$ et $95,4 \%$ de probabilité). Les intervalles à deux sigma, obtenus par la méthode de distribution des probabilités, sont ensuite présentés lorsqu'ils ont pu être calculés. Dans ce second cas, le chiffre entre parenthèse indique la contribution relative aux probabilités de chacun d'entre eux.

Les calibrations obtenues sont les suivantes (fig. 2) :

- SLP 25, fond de la fosse; Ly 6130 : $2845 \pm 40$ BP. Point moyen $=1001$ av. J.-C. Segments $=1046-926$ et 1187-901 av. J.-C;

SLE A11, remplissage de la fosse ; Ly 6119 : 3090 \pm 75 BP. Points moyens $=1388,1330,1323$ av. J.-C. Segments $=1431-1261$ et 1518-1128 av. J.-C. 1337$1372(100)$;

- SLE A2, fond de la fosse; Ly 6118 : $2865 \pm 65$ BP. Point moyen 1009 av. J.-C. Segments $=1187-924$ et 1259-839 av. J.-C. 1107-993 (100);

SLE A14, remplissage de la fosse : Ly 6117 : 2890 \pm 85 BP. Point moyen $=1047$ av. J.-C. Segments $1256-929$ et $1372-934$ av. J.-C. 1172-938 (100).

Il apparaît que la date de A11, avec ses points moyens situés en plein XIV $\mathrm{XV}^{\mathrm{e}}$ siècle, est plus ancienne d'au moins trois siècles que celles obtenues sur les autres structures. La typologie du mobilier céramique et la présence des fragments d'un même vase (fig. $5, \mathrm{n}^{\circ} 4$ ) dans cette fosse et dans A14 ne permettent pourtant pas de douter de sa contemporanéité avec l'ensemble. L'explication la plus plausible résiderait dans la combustion de bois anciens. Les trois autres datations sont par contre parfaitement cohérentes. La plage de recouvrement entre les intervalles s'établit entre 1046 et 929 av. J.-C. et les points moyens entre 1047 et 1001 . La plage de temps, calibrée à deux écart types, de la date de SLP 25, ne dépasse pas la fin du $\mathrm{X}^{\mathrm{e}}$ siècle. Il en est de même pour les fosses A2 et A14 des Estournelles si l'on considère le résultat obtenu suivant la méthode de distribution des probabilités. En prenant en compte que les dates ${ }^{14} \mathrm{C}$ ne peuvent représenter qu'un terminus post-quem (Vital dir., 1993, p. 35-36), il parât légitime d'envisager de dater ces structures entre la fin $\mathrm{du} \mathrm{XI^{ \textrm {e } }}$ et la première moitié du $\mathrm{X}^{\mathrm{e}}$ siècle av. J.-C.

Cette proposition est en phase avec plusieurs sites attribués au Bronze final IIIa ou au tout début du Bronze final IIIb :

- grotte du Gardon à Ambérieu-en-Bugey (Ain), couches 20-21. Deux dates ${ }^{14} \mathrm{C}$ réalisées dans ces niveaux donnent des points moyens de 1017 et 1005 av. J.-C. (fig. 2) et des intervalles de temps calibrés à deux sigma ne débordant pas sur le $\mathrm{IX}^{\mathrm{e}}$ siècle av. J.-C. (Voruz dir., 1993);

station de Tougues à Chens-sur-Léman (HauteSavoie), où les premières mesures dendrochronologiques effectuées sur des bois de l'ensemble 2 ont donné des dates d'abattage entre 1017 et 962 av. J.-C. (Billaud et Marguet, 1992);
- Chalon-sur-Saône, le Gué-des-Piles (Haute-Saône). Pour ce site, les derniers abattages sont datés de 956 av. J.-C. Une phase d'installation plus ancienne, dans les années 980-975 av. J.-C., est également probable. La dendrochronologie suggère donc fortement une durée d'occupation de l'ordre de trente ans (entre 980 et 950 av. J.-C. : Bonnamour et al., 1990);

- Hauterive-Champréveyres (Suisse, NE), zones D et E. Les constructions reconnues sur ce site sont datées de 981 à 961 av. J.-C. pour la zone D. Une date provisoire entre 908 et 873 av. J.-C. est proposée pour la zone E (Borrello, 1992, p. 23 et 48);

- Cortaillod-Est (Suisse, NE). Le centre de l'agglomération a été édifié entre 1010 et 964 av. J.-C. En périphérie, des abattages plus récents sont connus jusqu'en 955 av. J.-C. (Rychner et coll., 1988);

- Le Landeron (Suisse, NE) où trois phases d'abattage sont attestées en 961, 960 et 957 av. J.-C. (Rychner et coll., 1988);

- Vinelz-Ländti (Suisse, BE). Un pieu de la couche inférieure (couche 2) est daté de 1005 av. J.-C. La couche supérieure (couche 1) est quant à elle située postérieurement à 920 av. J.-C. par une datation ${ }^{14} \mathrm{C}$ (Gross, 1986).

LE MOBILIER CÉRAMIQUE
Choix méthodologiques et paramètres d'étude

Le mobilier présenté est issu de cinq fosses du site des Estournelles (A2, A10, A11, A12 et A14) et d'une structure de la Plaine (SLP 25). Cette dernière, avec un minimum de 131 individus identifiés, représente 56,7\% de l'ensemble étudié. La présence des fragments d'un même vase en A14 et A10, ainsi que l'homogénéité typologique et technologique des céramiques, autorisent une présentation descriptive globale de l'ensemble de la série. Le principal intérêt d'une démarche de ce type est de permettre d'éviter la répétition fastidieuse de caractérisations et de comparaisons analogues et d'autoriser une première approche quantifiée fondée sur un volume de matériel numériquement plus important. Elle n'exclut pas, de surcroît, la possibilité de tenter un travail d'analyse plus fin en confrontant chaque assemblage en termes de représentation de types identiques (cf. supra). Les associations par fosses sont, de toutes manières, conservées dans la présentation graphique des céramiques de chaque ensemble.

La classification et les dénominations retenues (qui ne revêtent aucun présupposé fonctionnel) sont fondées sur les paramètres d'analyse suivants, empruntés à diverses études concernant des collections régionales (Vital, 1990; Vital dir., 1993) :

- aplatissement général (rapport hauteur/diamètre maximum), permettant d'individualiser formes hautes (hauteur $\geq$ diamètre maximal) et formes basses (hauteur $<$ diamètre maximal);

- segmentation de la panse;

- orientation de la partie supérieure du vase par rapport à l'axe de symétrie;

- présence ou non d'un rebord. 


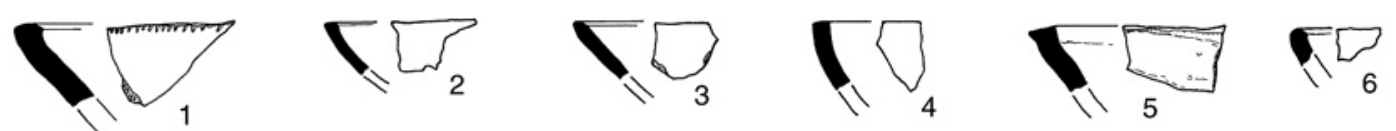

$$
\text { (2) }
$$

$\$ \sum_{10}$
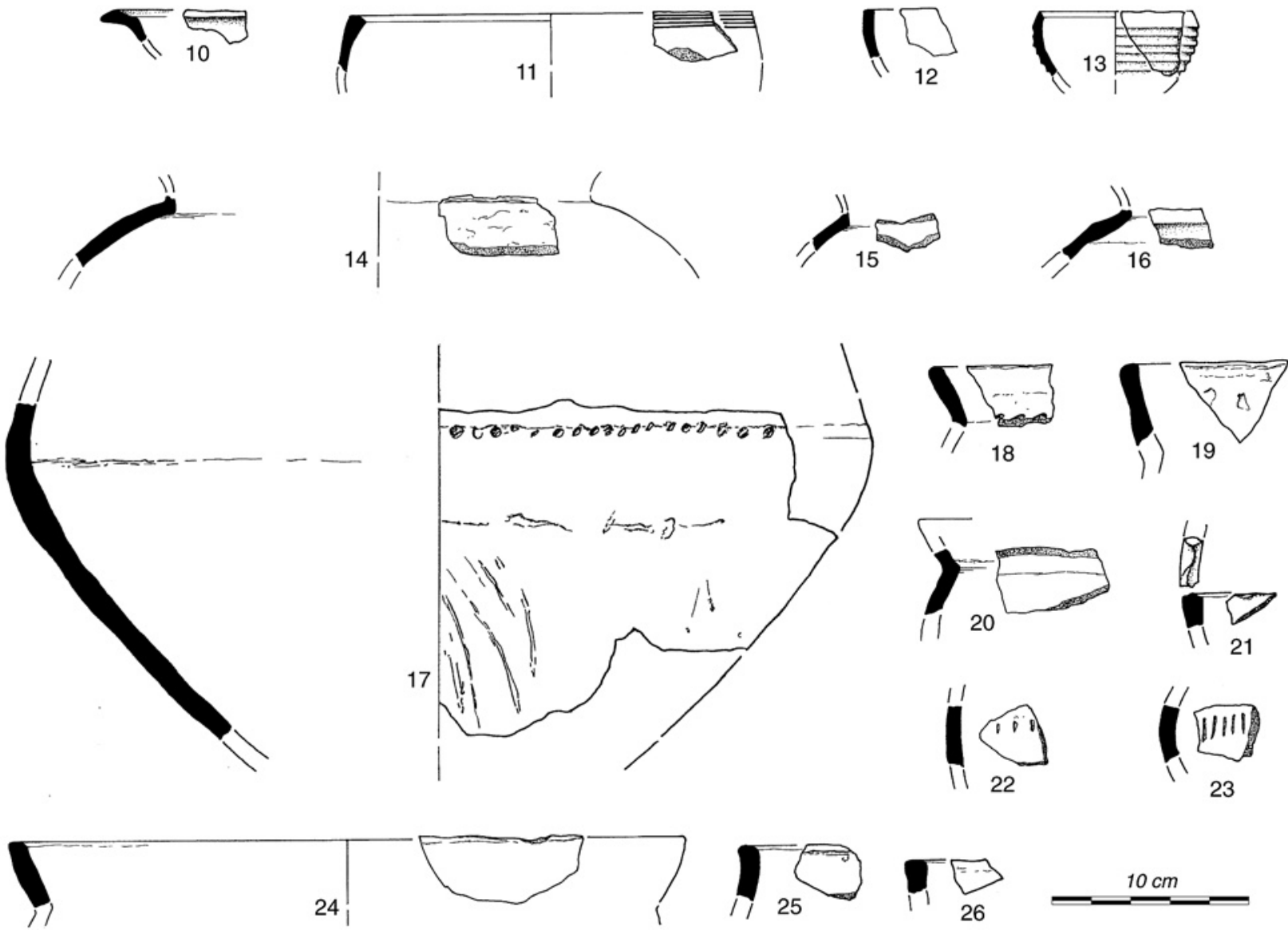

Fig. 3 - Mobilier céramique de la fosse A10 du site des Estournelles.

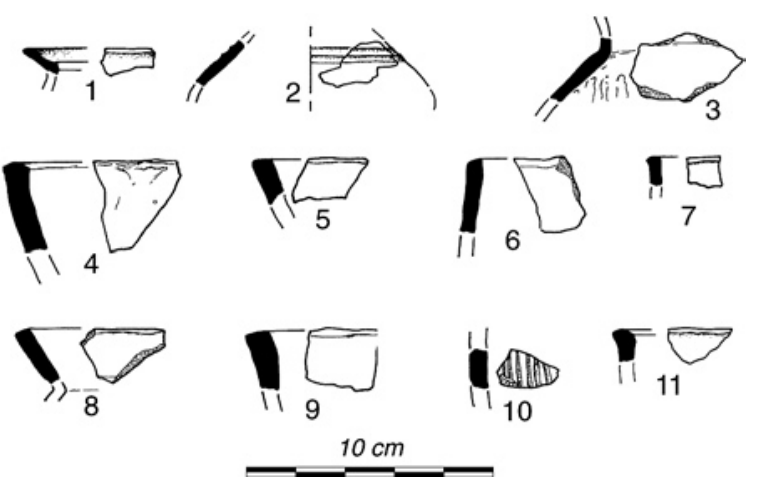

Fig. 4 - Mobilier céramique de la fosse A12 du site des Estournelles. 
Nous obtenons les types suivants :

- formes non segmentées; coupes et coupes à rebord;

- formes à segmentation unique; jattes et écuelles pour les formes basses, pots et jarres pour les formes hautes:

- formes à double segmentation; les mêmes que le groupe précédent avec le caractère cumulatif représenté par le rebord (soit : jattes à rebord, écuelles à rebord, pots à rebord et jarres à rebord);

- les gobelets sont des formes de petite dimension, à peu près aussi larges que hautes.

La définition de chacun de ces types est ensuite affinée par la prise en compte de particularités morphologiques ou décoratives spécifiques. Ces critères descriptifs présentent l'avantage de rester suffisamment souples et hiérarchisés pour permettre l'intégration d'une proportion importante de tessons dans le cas de séries fortement fragmentées.

En ce qui concerne les caractéristiques physiques et technologiques des pâtes et des céramiques, nous nous contenterons de retenir deux groupes définis visuellement :

- céramiques fines; dégraissant, de taille en général inférieure à $1 \mathrm{~mm}$ et traitement des surfaces très soigné (polissage...);

- céramique grossière; dégraissant pouvant atteindre ou dépasser une taille centimétrique et traitement de surface laissant apparaître des traces de montage et de façonnage.

Bien que la totalité des tessons présentant une caractéristique particulière (forme ou décor) soit représentée dans le catalogue, les décomptes proposés ne prendront en compte que les fragments attribuables avec certitude à un type donné, soit 216 vases sur un nombre minimum d'individus de 231. Enfin, nous n'avons pas relevé de différences prononcées entre le mobilier des Estournelles et de la Plaine, en partie trouvé en contexte funéraire, et celui des sites d'habitat mobilisés en comparaison.

\section{Les coupes}

Un total de soixante-quinze coupes a été reconnu, soit $34,72 \%$ de l'ensemble de la série. La prise de mesure des diamètres restituables définit deux grands ensembles de taille, le premier centré vers les $20 \mathrm{~cm}$, le second s'établissant entre 28 et $36 \mathrm{~cm}$. Un exemplaire de très grande dimension (diamètre de $47 \mathrm{~cm}$ ) est présent dans la fosse 25 (fig. 8, $\mathrm{n}^{\circ}$ 54). Un décompte effectué sur la forme des lèvres de 42 coupes en pâte fine montre une très forte majorité de lèvres chanfreinées (présentant au moins deux facettes, 20 exemplaires; fig. $8, \mathrm{n}^{\text {os }} 11,15,17$ et 21 , par exemple) et plates ou arrondies (17 exemplaires). Les lèvres biseautées ( 3 exemplaires : fig. $5, \mathrm{n}^{\text {os }} 1$ et 8 et fig. $8, \mathrm{n}^{\circ} 50$ ) ou ornées de cannelures fines ( 2 exemplaires, fig. $5, \mathrm{n}^{\circ}$ 5 et fig. $\left.7, n^{\circ} 2\right)$ sont par contre exceptionnelles. En ce qui concerne la forme générale de ces vases, nous observons que les profils concaves dominent largement l'ensemble (fig. 8, $\mathrm{n}^{\text {os }}$ 9, 10, 17 et 50 ). Les parois sont parfois très fines et montrent un épaississement marqué vers l'embouchure (fig. $5, \mathrm{n}^{\circ} 1$ et fig. $8, \mathrm{n}^{\text {os }} 17$ à 24 ). À l'exception d'une double ligne horizontale incisée près du fond et sur la face interne d'une coupe de SLP 25 (fig. 8, n 55), aucun décor n'a été observé, tant sur les tessons dessinés que sur les fragments de panse identifiés. Le caractère extrêmement ubiquiste de ces formes durant la fin de l'Âge du Bronze semble rendre caduque tout travail de comparaison. Cependant, trois caractéristiques de cette série appellent quelques remarques :

- la forte proportion de lèvres chanfreinées tranche avec ce qui est connu dans les séries bien documentées du Bronze final III de la région neuchâteloise (Borrello, 1986 et 1992 pour Cortaillod-Est et Hauterive-Champréveyres zone D, Bronze final IIIa; Rychner, 1979 pour Auvernier, Bronze final IIIb). Des coupes présentant cette caractéristique sont par contre présentes dans le sud du Jura sur le site de Saint-Alban locus B à Creys-et-Pusignieux (Isère; décapages 28 et 30, attribués à une phase récente du Bronze final IIIa; Treffort, 1993, pl. 28, n $^{\circ} 2$ et pl. $33, n^{\text {os }} 2$ à 4 et 6 ). La vérification de la valeur chronologique ou géographique de cette observation ne pourra se faire tant que nous ne disposerons pas de séries numériquement importantes;

- l'absence de décors incisés ou peignés (guirlandes, triangles hachurés, arêtes de poisson, motifs composites...) renvoie à ce qui a déjà été observé sur le mobilier des couches 18 à 20 de la grotte du Gardon à Ambérieu-en-Bugey, ou celui des niveaux inférieurs de Saint-Alban locus B (Ain; Voruz, 1991, p. 224 et Treffort, 1993) et semblerait confirmer cette particularité régionale vis-à-vis du Plateau suisse et de la Franche-Comté où ces ornements sont largement répandus (Borrello, 1986, pl. 15 à 33 et 1992, pl. 9 à 13, 48 et 49; grotte des Planches-près-Arbois horizon D2, Jura, Pétrequin et al., 1985, fig. 100, $\mathrm{n}^{\circ} 7$ et fig. 104, $\mathrm{n}^{\text {os }} 1$ et 2 );

- les décors de cannelures larges internes (ou gradins) sont également inconnus. Ce motif, largement représenté dans tout le domaine Rhin-Suisse-France orientale, est bien attesté localement durant le Bronze final IIb dans les sites de Vénissieux-le Vieux-Bourg (Rhône), de Tougues à Chens-sur-Léman (HauteSavoie), ensemble 3 et de la grotte des Gitans à Saint-Sorlin-en-Bugey dans l'Ain (Joly et Taras, 1994, pl. 4, n ${ }^{\circ}$, pl. 5, n ${ }^{\circ}$, pl. 12, n 3 et pl. 17, $\mathrm{n}^{\text {os }}$ 9, 11 et 12; Billaud et Marguet, 1992, fig. 22; Voruz, 1986, fig. 2, $\mathrm{n}^{\text {os }} 3$ et 4). Il semblerait devenir plus rare dès la première moitié du $\mathrm{X}^{\mathrm{e}}$ siècle av. J.-C. comme le montrent l'ensemble 3 de Tougues, les couches 18 à 20 du Gardon et Saint-Alban locus B. Quelques rares exemplaires résiduels sont également connus durant le Bronze final IIIb sur les sites de Montagnieu-le Pré de la Cour (Ain; Vital dir., 1993, fig. 59, $\mathrm{n}^{\circ}$ 6) et Saint-Alban locus A (Stahl, 1991, fig. $9, \mathrm{n}^{\text {os }} 3$ et 4 ). Il est totalement absent des sites de Châtillon à Chindrieux et des Barlières à Serrièresde-Briord datés du dernier quart du $\mathrm{IX}^{\mathrm{e}}$ siècle 

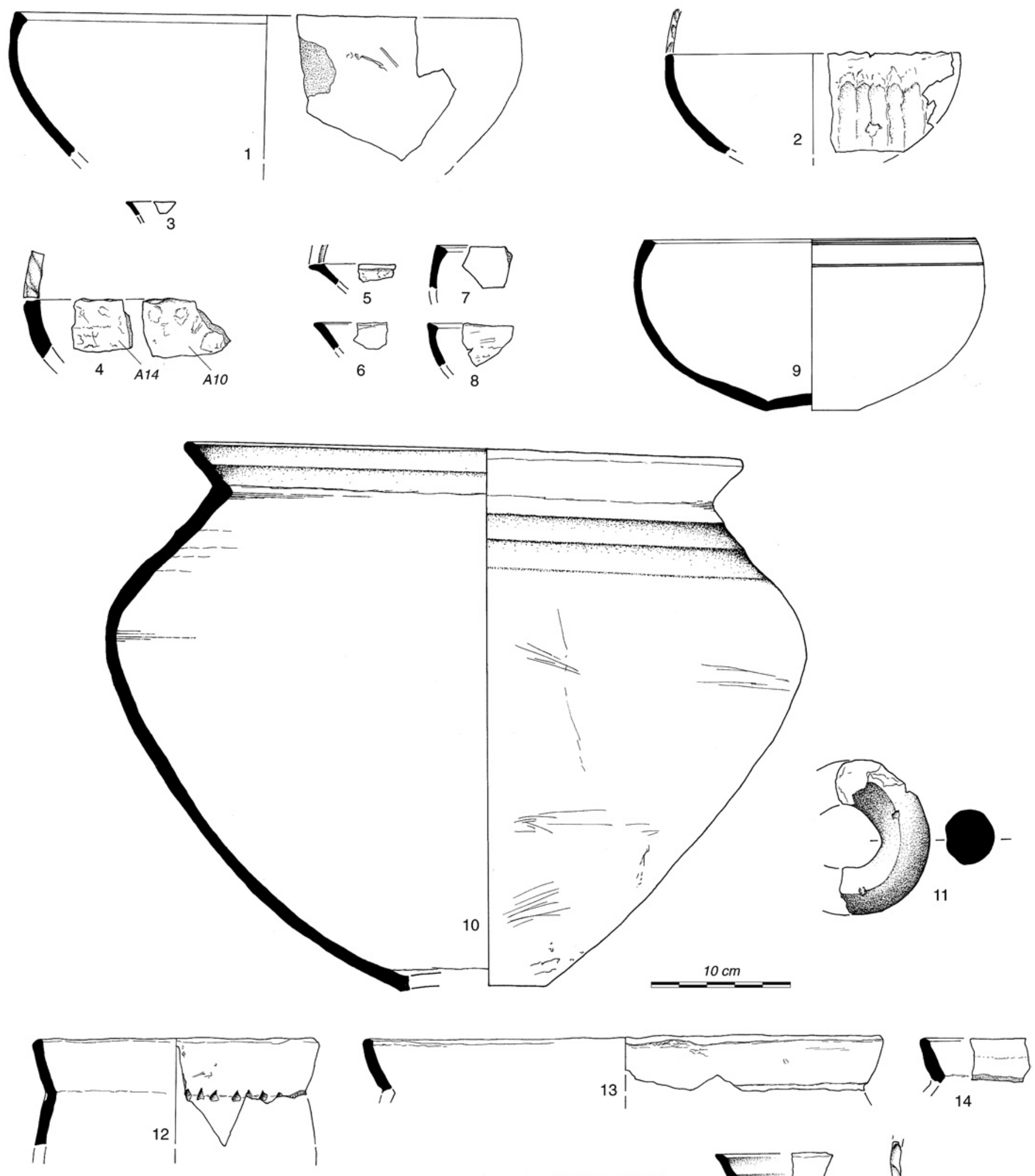

1
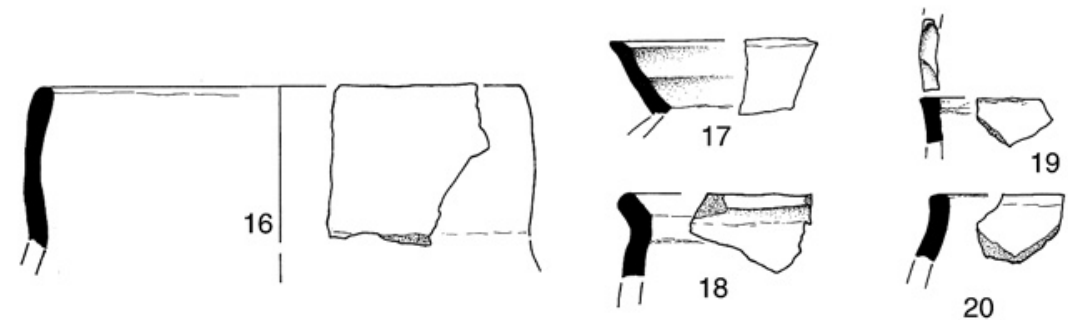

20

Fig. 5 - Mobilier céramique de la fosse A14 du site des Estournelles. 

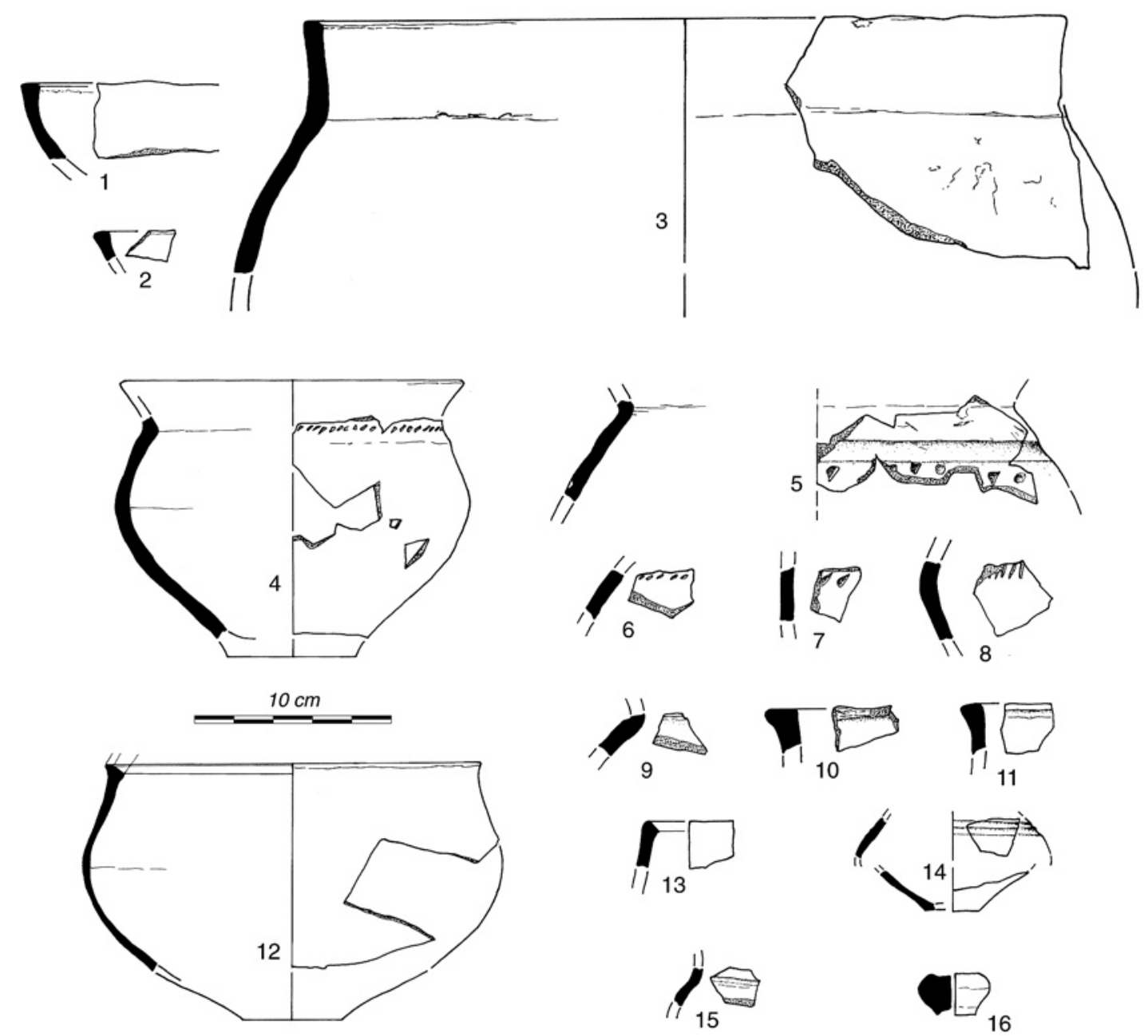

Fig. 6 - Mobilier céramique de la fosse A11 du site des Estournelles.

av. J.-C. (Savoie : Billaud et al., 1992; Ain : Thiériot, 2000). Le défaut de ce décor sur les coupes des Estournelles et de la Plaine pourrait donc s'inscrire dans ce schéma de disparition progressive. Notons enfin que ce motif est présent sur deux rebords de jarres de la fosse A14 (fig. 5, $\mathrm{n}^{\text {os }} 10$ et 17).

Les coupes en pâte grossière sont minoritaires. Leurs lèvres sont parfois digitées (fig. $5, \mathrm{n}^{\text {os }} 2$ et 4 ). Un exemplaire à large fond (fig. 8, n० 49) trouve une comparaison directe dans le décapage 29 de Saint-Alban locus B (Treffort, 1993, fig. 28, n 3 ).

\section{Les coupes à rebord}

Huit vases de ce type, non décorés, ont été reconnus (soit $3,70 \%$; fig. $3, \mathrm{n}^{\circ} 10$ et fig. $8, \mathrm{n}^{\text {os }} 1$ à 7 ). Cette forme connaît une large répartition régionale durant le Bronze final IIIb : Jura méridional (les Barlières : Thiériot, 2000, pl. 5, no 19 et pl. 37, $\mathrm{n}^{\text {os }} 232$ à 235), moyenne vallée du Rhône (les Gandus à Saint-FerréolTrente-Pas, Drôme : Daumas et Laudet, 1985, fig. 9; baume des Anges à Donzère, Drôme: Vital, 1990, fig. $41, \mathrm{n}^{\text {os }} 1$ à 3 ), domaine péri-alpin (Balme de Glos à Sassenage, Isère : Bocquet, 1969, fig. 51, n 16; station de Châtillon à Chindrieux, Savoie : Billaud et al., 1992, fig. 8, $\mathrm{n}^{\circ} 2$ ).

Sa présence en plusieurs exemplaires est également attestée en contexte Bronze final IIIa au Gardon, à Saint-Alban locus B, dans la fosse 19 du site ZAC des Perches locus 2 dans le Rhône et à la grotte des Cloches à Saint-Martin-d'Ardèche dans l'Ardèche (Voruz, 1991, fig. 94, $\mathrm{n}^{\circ} 3$ et fig. 97, $\mathrm{n}^{\circ} 2$; Treffort, 1993, fig. $37, \mathrm{n}^{\text {os }} 1$ et 2 ; Hénon, 1999 , fig. $46, \mathrm{n}^{\text {os }} 1$ et 2 ; Vital, 1987, fig. $14, \mathrm{n}^{\text {os }} 1,2,4$ et 6 ). Une situation analogue se retrouve en Languedoc oriental, où des formes identiques sont connues dès le Bronze final IIIa (grotte I de Castelviehl à Sainte-Anastasie couche C6, Gard : Dedet et Pène, 1991, fig. 20 et $21, \mathrm{n}^{\circ} 1$ ) et jusqu' au Bronze final IIIb (site de Lombren à Vénéjan, Gard : Dedet et Charmasson, 1989, fig. 10, nos 1 à 7 ; oppidum de Triple-Levée à Beaucaire, Gard : Dedet et al., 1978, fig. 10, $\mathrm{n}^{\circ} 16$ ). Elles sont, par contre, exceptionnelles sur le Plateau suisse, où un exemplaire décoré de motifs géométriques gravés organisés en métopes concentriques est présent dans la série de Mörigen et daté de la fin du Bronze final IIIb (Bernatzky-Goetze, 1987, Taf. $55, \mathrm{n}^{\circ} 1$ ). Une ébauche de schéma géographique 


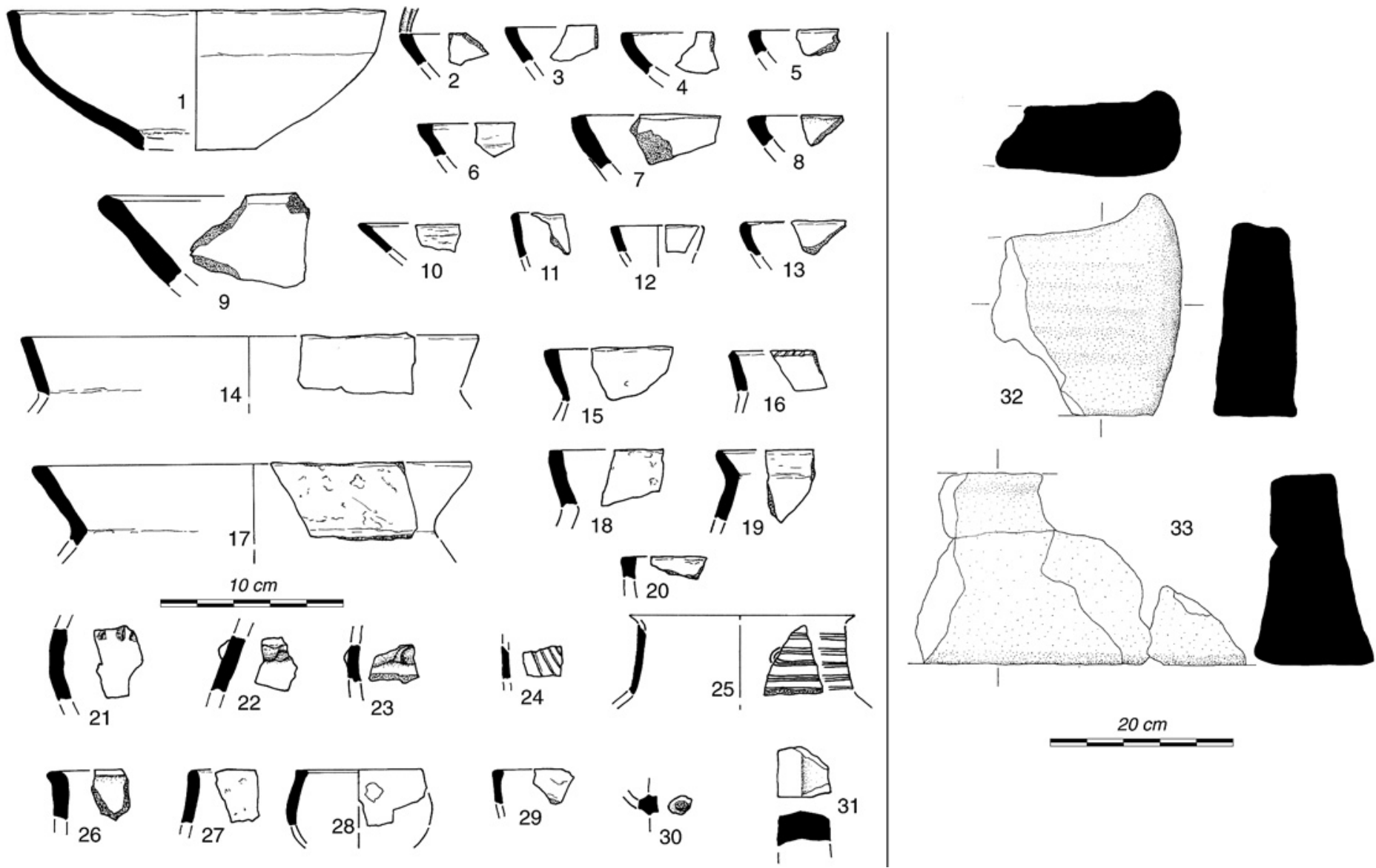

Fig. 7 - Mobilier céramique de la fosse A2 du site des Estournelles ( $\mathrm{n}^{\text {os }} 1$ à 31 ) et chenets de la fosse 25 du site de la Plaine (n ${ }^{\text {os }} 32$ et 33 , dessins F. Cordier, INRAP).

pourrait donc se dessiner. Néanmoins le problème de l'origine locale ou méridionale de cette forme ne peut être résolu pour l'instant.

\section{Les jattes}

Vingt-six vases en pâte fine entrent dans cette catégorie $(12,04 \%)$. La forme de la partie supérieure permet de distinguer deux groupes :

- jattes à profil arrondi et partie supérieure rectiligne, parfois marquée par un épaississement sensible vers l'embouchure (fig. $3, \mathrm{n}^{\circ} 11$; fig. $5, \mathrm{n}^{\text {os }} 7$ et 9 et fig. 9 , $\mathrm{n}^{\text {os }} 2,5$ et 6$)$. Les lèvres sont le plus souvent chanfreinées ( 8 cas sur 11 ; fig. $3, \mathrm{n}^{\circ} 11$, fig. $5, \mathrm{n}^{\text {os }} 7$ et 9 , fig. $\left.9, \mathrm{n}^{\circ} 2 \ldots\right)$, plus rarement biseautées (3 exemplaires : fig. $5, \mathrm{n}^{\circ} 1$, fig. $6, \mathrm{n}^{\circ} 13$ et fig. $9, \mathrm{n}^{\circ} 8$ ). Les décors reconnus sur trois vases consistent en une série de lignes incisées horizontales situées juste sous la lèvre (fig. $3, n^{\circ} 11$ ) pouvant être associée à un double filet sur la partie supérieure du vase (fig. 5, $\mathrm{n}^{\circ} 9$ ) et à des cannelures fines soulignant le point d'inflexion de la panse (fig. 9, $\mathrm{n}^{\circ} 5$ ). Ce type de forme est bien représenté régionalement dans des séries datées ou attribuées au Bronze final IIIa: grotte du Gardon (Voruz, 1991, fig. 93, nos 2 et 3), Saint-Alban locus B (Treffort, 1993, fig. 32, $\mathrm{n}^{\circ} 2$ ), Balme à Gontran à Chaley (Ain; Treffort, Nicod et coll., 2000, fig. 14, $\mathrm{n}^{\text {os }} 13$ à 15), station de Tougues (Billaud et Marguet, 1992, fig. 23), Saint-Priest, ZAC des Perches locus 2, structure 24 (Hénon, 1999, fig. 49, $\mathrm{n}^{\circ} 7$ ), grotte des Cloches à Saint-Martind'Ardèche (Ardèche; Vital, 1987, fig. 11, n ${ }^{\text {os }} 10$ et 11). Elles connaissent également une répartition géographique assez large : Val de Saône (site du Gué-des-Piles à Châlon-sur-Saône, Saône-et-Loire; Bonnamour, 1989, fig. 20, $\mathrm{n}^{\circ}$ 13), Franche-Comté (grotte des Planches-près-Arbois horizon D2; Pétrequin et al., 1985, fig. 109, $\mathrm{n}^{\text {os }} 1$ et 3 ), Languedoc (grotte I de Castelviehl à Sainte-Anastasie couche B3; Dedet et Pène, 1991, fig. 23, n 10, marquées par un épaississement de la partie supérieure identique à celui de certains exemplaires des Estournelles et de la Plaine). Ce type est également connu en Suisse occidentale. Très fréquent à Cortaillod-Est et Hauterive-Champréveyres zone D (Bronze final IIIa) avec des décors de lignes horizontales en registre ou de méandres symétriques, il devient plus rare à Hauterive zone E (Borrello, 1986 et 1992). Les quelques exemplaires attestés à Auvernier sont attribués soit au Bronze final IIIa soit au Bronze final III sans précision (Rychner, 1979, pl. 19 à 21). Notons en dernier lieu que cette forme est inconnue à Hauterive-Champréveyres zones A et B (Bronze final IIb évolué : Borrello, 1993; Rychner, 1988, p. 126-127). 
L'évolution perceptible dans cette région peut permettre de considérer ce type de forme comme caractéristique du $\mathrm{X}^{\mathrm{e}}$ siècle av. J.-C.;

- trois jattes sont caractérisées par une paroi très fine et par une lèvre chanfreinée épaissie (fig. $6, \mathrm{n}^{\circ} 12$ et fig. 9, $\mathrm{n}^{\text {os }} 1$ et 6). Elles trouvent comparaison dans des contextes de la première moitié du $\mathrm{X}^{\mathrm{e}}$ siècle à Tougues ensemble 2 (Billaud et Marguet, 1992, fig. 23), Saint-Alban locus B, décapage 36 (Treffort,
1993, fig. 43, n 7). Ce type est représenté à la Balme à Gontran et dans la fosse 19 de la ZAC des Perches locus 2 (Treffort, Nicod et coll., 2000, fig. 14, $\mathrm{n}^{\circ} 2$; Hénon, 1999, fig. 19, ${ }^{\circ}$ 6). Un exemplaire proche est également connu dans la tombe 2 du site de Lausanne-Vidy attribuée par une date ${ }^{14} \mathrm{C}$ et la typologie du mobilier au Bronze final IIIa (Kaenel et Klausener, 1990, pl. 2, $\left.\mathrm{n}^{\circ} 10\right)$. Un vase de ce type trouvé à Hauterive-Champréveyres zone E serait l'un
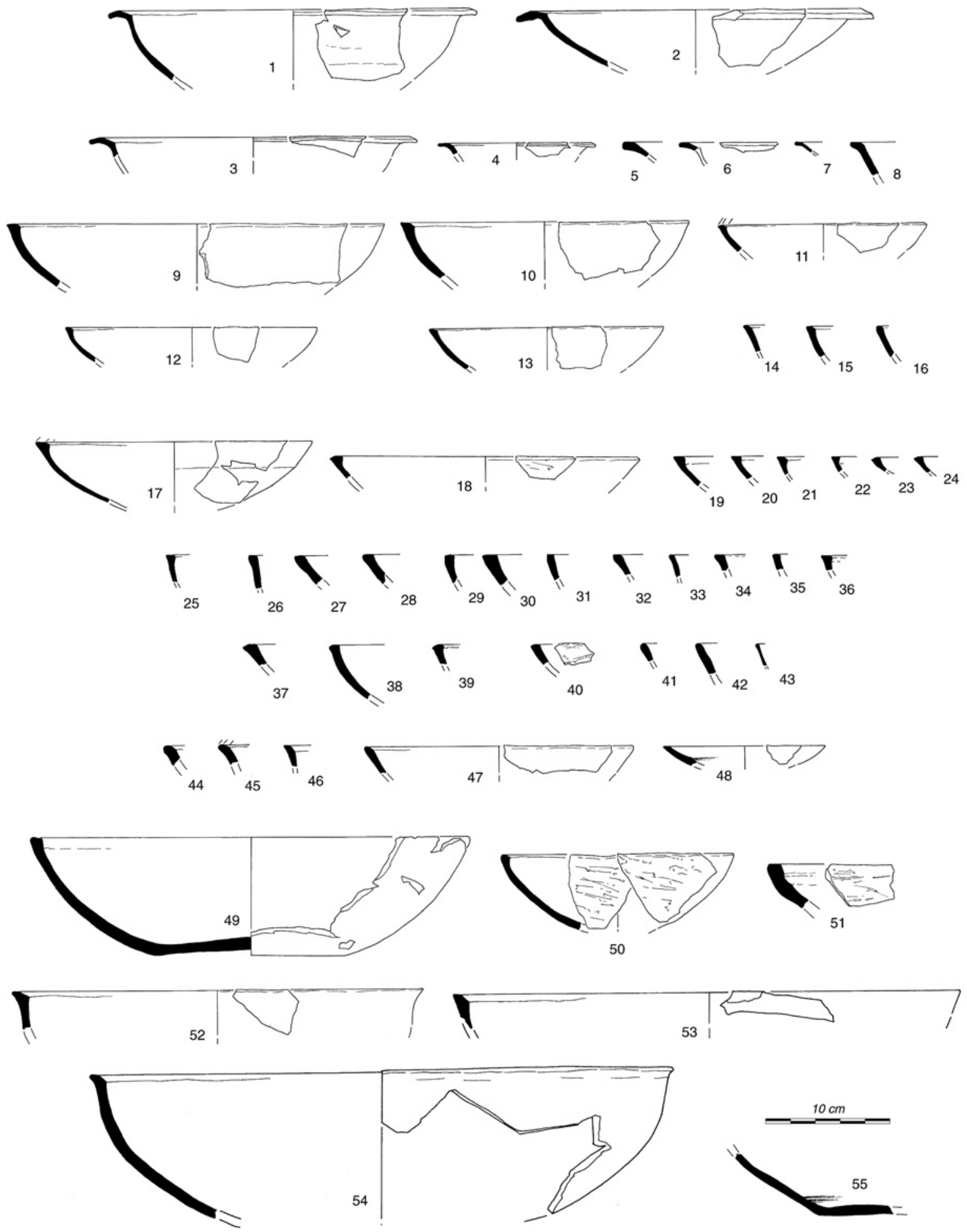

Fig. 8 - Mobilier céramique de la fosse 25 du site de la Plaine. 


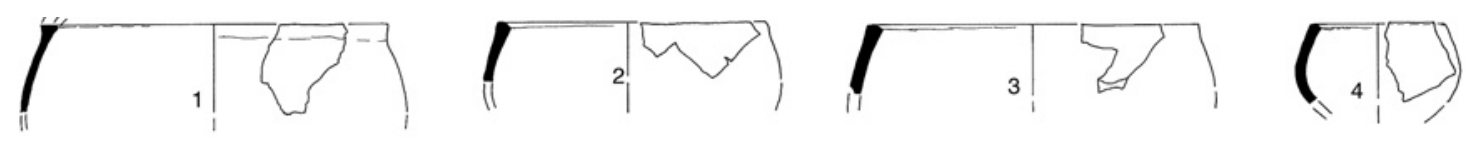

I. 四四 C.
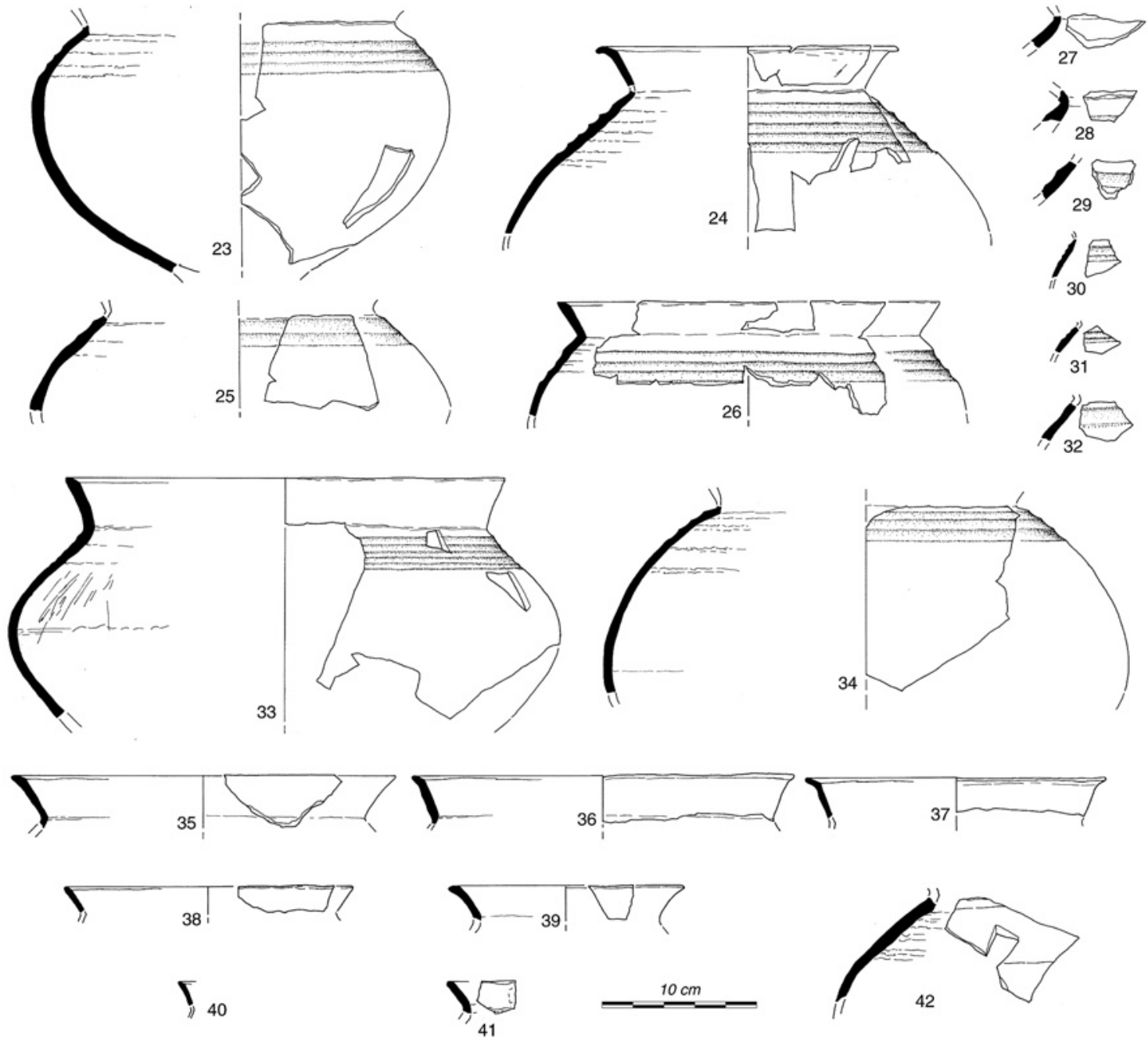

Fig. 9 - Mobilier céramique de la fosse 25 du site de la Plaine. 
des plus récents (début du Bronze final IIIb; Borrello, 1992, pl. 51, n 6 ).

\section{Les jarres à rebord}

Ce groupe est constitué de vingt-quatre vases en pâte fine, soit $14,35 \%$ de l'ensemble. Leurs rebords sont déversés, courts et portent le plus souvent une lèvre chanfreinée (huit cas sur onze; fig. 5, ${ }^{\circ} 17$, fig. 9, $\mathrm{n}^{\mathrm{os}} 33,35$ et $\left.36 \ldots\right)$. Sur les vingt et un fragments de panse identifiés, quinze portent un décor appliqué sur la partie supérieure du corps du vase. Il est formé le plus souvent d'une ou plusieurs cannelures horizontales fines ou larges (fig. $5, \mathrm{n}^{\circ} 10$, fig. $9, \mathrm{n}^{\text {os }} 20$ et 22 à $26 \ldots$ ). Un cas unique de motif associant cannelures, séries de doubles lignes incisées et guillochis est attesté (fig. 9, $n^{\circ} 21$ ). Les exemplaires les mieux conservés autorisent l'identification de deux groupes définis suivant le profil général des vases : formes arrondies (fig. $9, \mathrm{n}^{\text {os }} 21$, $23,34 \ldots$ ) ou anguleuses (fig. $5, \mathrm{n}^{\circ} 10$, fig. $9, \mathrm{n}^{\text {os }} 20$ et 33). La fragmentation de la série ne permet malheureusement pas un décompte fiable entre ces deux ensembles.

Ce type de vase est extrêmement fréquent dans les sites du Bronze final IIIlb du nord de la région Rhône-Alpes : les Barlières et Saint-Alban locus A et B pour le sud du Jura (Thiériot, 2000, pl. 11 à 14; Stahl, 1991, pl. 6 et 7; Treffort, 1993, pl. 19, nos 4,6 à 9,11 , fig. $21, n^{\text {os }} 2$ à 5...), l'ensemble 1 de Tougues ou la station de Chindrieux pour les lacs savoyards (Billaud et Marguet, 1992, fig. 24; Billaud et al., 1992, fig. 12, $\mathrm{n}^{\text {os }}$ 6, 7 et 10). Il est également connu dans la moyenne vallée du Rhône français, souvent marqué par un aplatissement de la partie supérieure à proximité de la jonction panse/rebord : baume des Anges à Donzère, Drôme (Vital, 1990, fig. $48, n^{\text {os }} 10$ à 12 , fig. $49, n^{\text {os }} 1$ à 19 ), les Gandus (Daumas et Laudet, 1985). Nous envisageons cependant de situer son apparition dans le courant du $\mathrm{X}^{\mathrm{e}}$ siècle, voire antérieurement. En témoignent les exemplaires des décapages 33 à 36 de Saint-Alban locus B attribués à la fin du Bronze final IIIa (Treffort, 1993, fig. 40, $\mathrm{n}^{\text {os }} 2$ et 6, fig. $41, n^{\circ} 2$ et fig. $45, n^{\circ} 6$ ), ou ceux du Gué-des-Piles datés du milieu du X $\mathrm{X}^{\mathrm{e}}$ siècle (Bonnamour, 1989, fig. 19, $\mathrm{n}^{\text {os }} 1$ et 2 ). Une situation différente semble perceptible pour le Plateau suisse. À Vinelz-Ländti, sur le lac de Bienne, les vases de ce type n'apparaissent que dans la couche supérieure pour laquelle une datation postérieure à 920 av. J.-C. est proposée (Gross, 1986, p. 33 et fig. 16). Il en est de même à Hauterive-Champréveyres où ils ne sont connus que dans la zone $\mathrm{E}$, la plus récente du site (cf. supra, Borrello, 1992, pl. 55, nº 1).

Si la documentation régionale ne permet pas encore de définir des caractères pouvant revêtir une signification chronologique pour ce type de forme, quelques particularités propres aux Estournelles et à la Plaine méritent d'être soulignées :

- les larges cannelures internes attestées sur deux rebords (fig. $5, \mathrm{n}^{\text {os }} 10$ et 17 ) sont inconnues sur ce type de vases dans les séries régionales du Bronze final IIIb. Une évolution similaire à celle des coupes portant ce motif doit être envisagée (cf. supra);
- une jarre à rebord déversé présentant un profil biconique accusé et une embouchure nettement rétrécie (fig. $9, \mathrm{n}^{\circ} 24$ ) trouve des comparaisons proches dans des ensembles datés du Bronze final IIb ou IIIa : Vénissieux-le Vieux-Bourg, fosse A96, grotte des Planches ou gisement $\mathrm{C}$ des Basses-Godernes à Champagne-sur-Seine par exemple (Joly et Taras, 1994, pl. 26, n 1; Pétrequin et al., 1985, fig. 122, $\mathrm{n}^{\circ} 1$; Senée, 1988, fig. $5, \mathrm{n}^{\circ}$ 9). Ces caractéristiques semblent par contre inconnues dans les ensembles du IX ${ }^{e}$ siècle av. J.-C;

- un exemplaire de petites dimensions à profil biconique et ouverture rétrécie (fig. $9, \mathrm{n}^{\circ} 20$ ) appelle les mêmes observations. Des formes identique décorées de cannelures larges ou d'incisions horizontales sont connues dans l'abri du Cuze à Sainte-Anastasie, daté du Bronze final IIb ou IIIa, et dans la série Bronze final IIIa de la grotte des Cloches (Cantal : Daugas et Vital, 1988, fig. 8, n 3; Ardèche : Vital, 1987, fig. $\left.12, \mathrm{n}^{\circ} 17\right)$. La couche 17 de la grotte du Gardon a livré un vase en céramique grossière présentant un profil très proche (Bronze final IIIa; Voruz, 1991, fig. $\left.97, \mathrm{n}^{\circ} 1\right)$.

\section{Les gobelets}

\section{Les gobelets ovoïdes}

Un exemplaire de la fosse 25 (fig. 11, $\mathrm{n}^{\circ} 7$ ), aussi large que haut, porte un court rebord déversé et est décoré de trois cannelures fines horizontales. Deux fragments de rebord (fig. $9, \mathrm{n}^{\text {os }} 12$ et 13 ) pourraient appartenir à ce type. Cette forme semble apparaitre au moins dès la première moitié du $\mathrm{X}^{\mathrm{e}}$ siècle av. J.-C., comme l'atteste sa présence dans la station de Tougues ou dans la grotte des Planches (Billaud et Marguet, 1992, fig. 23; Pétrequin et al., 1985, fig. 115, n ${ }^{\circ}$ ). Un vase au profil similaire d'Hauterive-Champréveyres zone D, dont la datation est très proche de celle de Tougues ensemble 2, confirmerait cette observation (Borrello, 1992, pl. 31, n 8). Un exemplaire du Gué-des-Piles porte un décor de méandres symétriques (Bonnamour, 1989, fig. 19, n 4).

Les comparaisons avec les séries régionales du Bronze final IIIb sont par contre beaucoup moins convaincantes, les nombreux gobelets de cette phase présentant presque systématiquement un profil caractéristique nettement plus surbaissé (les Barlières : Thiériot, 2000, pl. $10, \mathrm{n}^{\text {os }} 68$ à 70 et 72 à 78 ; Saint-Alban locus B, décapages 16 à 21 : Treffort, 1993, fig. 62). Les exemplaires les plus anciens de ce type de vase sont, dans l'état actuel de la documentation, ceux de Tougues, ensemble 1 (vers - 905 : Billaud et Marguet, 1992, fig. 24).

\section{Les gobelets à épaulement}

Cette forme est exceptionnelle et ne semble représentée avec certitude que par un fragment de col légèrement rentrant portant un motif de méandres symétriques réalisé au peigne à dents souples (fig. $7, \mathrm{n}^{\circ} 25$ ). Plusieurs autres tessons pourraient néanmoins relever de 


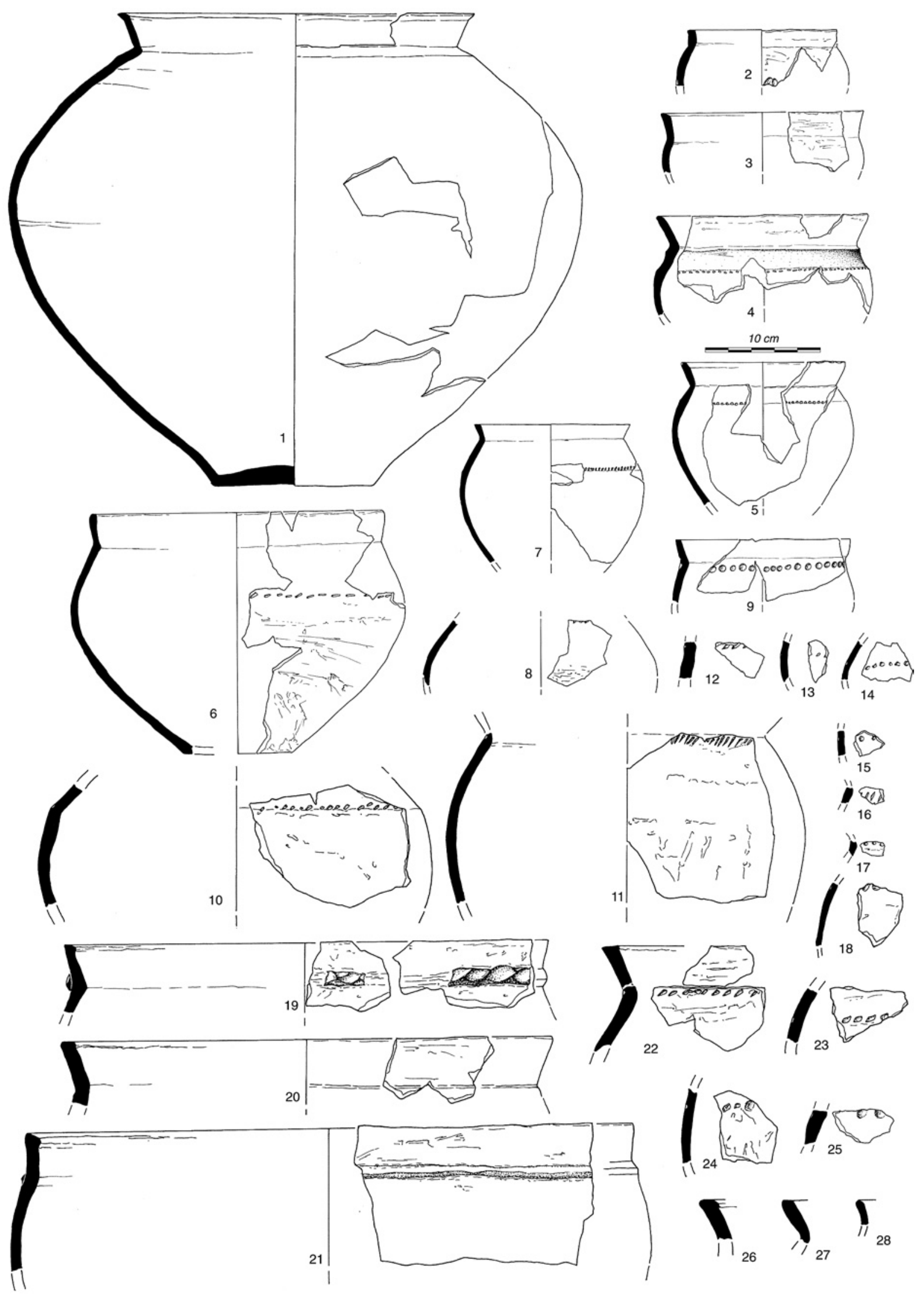

Fig. 10 - Mobilier céramique de la fosse 25 du site de la Plaine. 
ce groupe : deux fragments d'épaule portant un décor cannelé (fig. $4, n^{\circ} 2$ et fig. $6, n^{\circ} 14$ ) ainsi qu'une petite "coupelle" à fond étroit dont la lèvre semble porter des traces d'abrasion permettant d'envisager le possible réemploi d'une partie inférieure de gobelet après bris du vase (fig. $9, \mathrm{n}^{\circ} 16$ ). Ce type est extrêmement courant dans les séries du Bronze final IIb de la haute vallée du Rhône français : tombe 24 de la nécropole de la Croix Tombée à Pérouges, Ain (Vital, 1988, fig. 3, $\mathrm{n}^{\circ} 4$ ), Vénissieux-le Vieux-Bourg, Rhône (fosses 189, 94, 96; Joly et Taras, 1994, pl. 5, nº 6, fig. 10, $\mathrm{n}^{\text {os }} 1$ et 2, fig. 17, $\mathrm{n}^{\circ}$ 3) grotte de la Balme à La Balme-lesGrottes, Isère (Bocquet et Reymond, 1976, fig. 80, $\mathrm{n}^{\circ} 4$ ), station de Tougues, ensemble 3 (Billaud et Marguet, 1992, fig. 22). Elle est également connue durant la première moitié du $\mathrm{X}^{\mathrm{e}}$ siècle av. J.-C., comme l'atteste le mobilier de l'ensemble 2 de ce même site (Billaud et Marguet, 1992, fig. 23). À la grotte du Gardon, les gobelets à épaulement sont connus par quelques rares fragments issus des fouilles anciennes (Dumont et Treffort, 1991, fig. 18, $\mathrm{n}^{\circ}$ 2). Les travaux récents en ont également livré quelques exemplaires (inédit, renseignement J.-L. Voruz et J.-M. Treffort). La documentation régionale ne permet pas encore d'envisager une évolution typochronologique de la forme générale de ces récipients. Il apparaît néanmoins que le profil rentrant du col de gobelet de la fosse A2 trouve des parallèles dans les exemplaires les plus anciens : ensemble 3 de Tougues, nécropole de la Croix Tombée (Billaud et Marguet, 1992, fig. 22 ; Vital, 1988, fig. 3, n $\left.{ }^{\circ} 4\right)$. D'une manière plus générale, leur fréquence dans les séries semble diminuer durant le $\mathrm{X}^{\mathrm{e}}$ siècle.

\section{Les pots à rebord}

Un nombre minimal de soixante-sept individus de ce type a été reconnu (31,02\%). Bien que la série soit très fragmentée, les exemplaires les mieux conservés montrent le plus souvent des profils à panse arrondie dont le point d'inflexion est souvent situé dans le tiers supérieur du vase (fig. 10, $\mathrm{n}^{\text {os }} 2,4,5$ et 7 , fig. $11, \mathrm{n}^{\text {os }} 1$, 2 et 6). La jonction panse/rebord est en général bien marquée, parfois soulignée par une petite gorge (fig. 10, $\mathrm{n}^{\text {os }} 2$ et 5 , fig. 9 et fig. $11, \mathrm{n}^{\circ} 4$ ). Vingt-quatre de ces vases ont pu faire l'objet d'une mesure du diamètre à l'ouverture : six d'entre elles sont strictement inférieures à $20 \mathrm{~cm}$, sept sont comprises entre 20 et $28 \mathrm{~cm}$, six entre 30 et $34 \mathrm{~cm}$ et cinq sont supérieures à $36 \mathrm{~cm}$. Hormis un exemplaire de petite dimension (fig. 10, $\mathrm{n}^{\circ} 7$ ), ces vases sont systématiquement façonnés en pâte grossière. Des décomptes effectués sur la forme des rebords, les décors et leur position sur les vases donnent les résultats suivants :

- sur un total de trente-neuf rebords, vingt présentent un profil cintré concave peu marqué (fig. $5, \mathrm{n}^{\circ} 12$, fig. $10, \mathrm{n}^{\text {os }} 6$ et $19 \ldots$ ) ou accentué (fig. $11, \mathrm{n}^{\text {os }} 2$ et $3 . .$.$) pour dix-neuf rectilignes. Seulement quatre$ lèvres sont décorées (trois motifs digités : fig. 3, $\mathrm{n}^{\circ} 21$, fig. $5, \mathrm{n}^{\circ} 19$ et fig. $10, \mathrm{n}^{\circ} 5$; un cas de courtes lignes obliques appliquées à l'extérieur de la lèvre : fig. $\left.7, \mathrm{n}^{\circ} 16\right)$;
- les décors impressionnés ou estampés sont les plus fréquents (32 cas sur 40). Ils sont majoritairement réalisés à l'aide d'un outil (baguette, couteau, tête d'épingle; fig. $\left.10, \mathrm{n}^{\circ} 9 \ldots\right)$, beaucoup plus rarement digités (fig. $10, \mathrm{n}^{\circ} 2$, fig. $11, \mathrm{n}^{\text {os }} 2$ et 6 ). Des cordons impressionnés ou lisses sont appliqués sur quatre vases (fig. 7, $\mathrm{n}^{\text {os }} 22$ et 23, fig. 10, $\mathrm{n}^{\text {os }} 19$ et 21 ). Enfin, des motifs associant impressions et cannelures sont connus dans quatre cas (fig. 6, $\mathrm{n}^{\circ} 5$, fig. 10, $\mathrm{n}^{\circ} 4$, fig. $11, \mathrm{n}^{\text {os }} 1$ et 6$)$;

- ces décors sont situés soit sur la carène ou sur la partie supérieure du corps des vases, soit à la jonction panse/rebord (respectivement 23 et 13 exemplaires). Deux vases portent une ligne horizontale d'impressions à la base du rebord et une cannelure large sur l'épaule (fig. $11, \mathrm{n}^{\text {os }} 1$ et 6 ).

Ce type de forme est, d'une manière générale, bien représenté durant une période comprise entre le Bronze final IIb et le Bronze final IIIb dans la moitié nord de la région Rhône-Alpes :

- sites de Venissieux-le Vieux-Bourg, fosses 156, 94, 96... (Joly et Taras, 1994, pl. 2, n ${ }^{\circ}$, pl. 3, n 1, pl. 9, $\mathrm{n}^{\circ} 1$, pl. $10, \mathrm{n}^{\circ} 6$, pl. $22, \mathrm{n}^{\text {os }} 1,5,7$ et $\left.8 \ldots\right)$ et de Pommiers-en-Forez - la Maniriat dans la Loire (Daugas et Vital, 1988, fig. 4, n ${ }^{\text {os }} 1$ à 5) pour le Bronze final IIb.

- Grotte du Gardon, couches 18 à 20 (Voruz, 1991, fig. 95, $\mathrm{n}^{\text {os }} 1$, 3 et 4), Saint-Alban, locus B, décapages 34 à 37 (Treffort, 1993, fig. 40, $\mathrm{n}^{\text {os }} 8$ et 9, fig. 42, $\mathrm{n}^{\circ} 11$ et fig. $45, \mathrm{n}^{\text {os }} 3$ et 13 ) et Tougues, ensemble 2 (Billaud et Marguet, 1992, fig. 23) pour le Bronze final IIIa.

Sites de Montagnieu-le Pré de la Cour (Vital dir., 1993, fig. 57, 60, 63, $\mathrm{n}^{\circ} 11$ et fig. 64, $\mathrm{n}^{\text {os }} 2$ et 4 par exemple) et des Barlières (Thiériot, 2000, pl. 20 à $22 \ldots$ ) pour le Bronze final IIIb.

S'il semble à l'heure actuelle difficile de saisir une évolution dans le profil général de ce groupe de céramique pendant la deuxième moitié du Bronze final, la fréquence des cols cintrés concaves ainsi que celle des décors appliqués soit sur la carène, soit sur la partie supérieure du corps des vases, témoignent d'une progression au cours des $\mathrm{X}^{\mathrm{e}}$ et $\mathrm{IX}^{\mathrm{e}}$ siècles av. J.-C. Ces caractéristiques, isolées ou associées, sont très courantes sur les pots à rebord du site de Vénissieux-le Vieux-Bourg (Joly et Taras, 1994). Nous avons vu plus haut qu'elles sont présentes dans des proportions importantes sur les sites des Estournelles et de la Plaine (près de la moitié des rebords sont concaves; les décors sur carène ou épaulement constituent près des deux tiers de la série). Ils deviennent beaucoup plus rares durant la première moitié du $\mathrm{IX}^{\mathrm{e}}$ siècle (quelques exemplaires connus au Pré de la Cour; Vital dir., 1993, fig. $60,63, n^{\circ} 7$ et fig. $67, n^{\circ} 21$ ), pour disparaître complètement à la fin de celui-ci (site des Barlières; Thiériot, 2000).

L'association cannelures-impressions, minoritaire dans les deux sites de Simandres, est également un critère inconnu ou très rare dans les séries Bronze final IIIb de la haute vallée du Rhône français. Il est par contre 

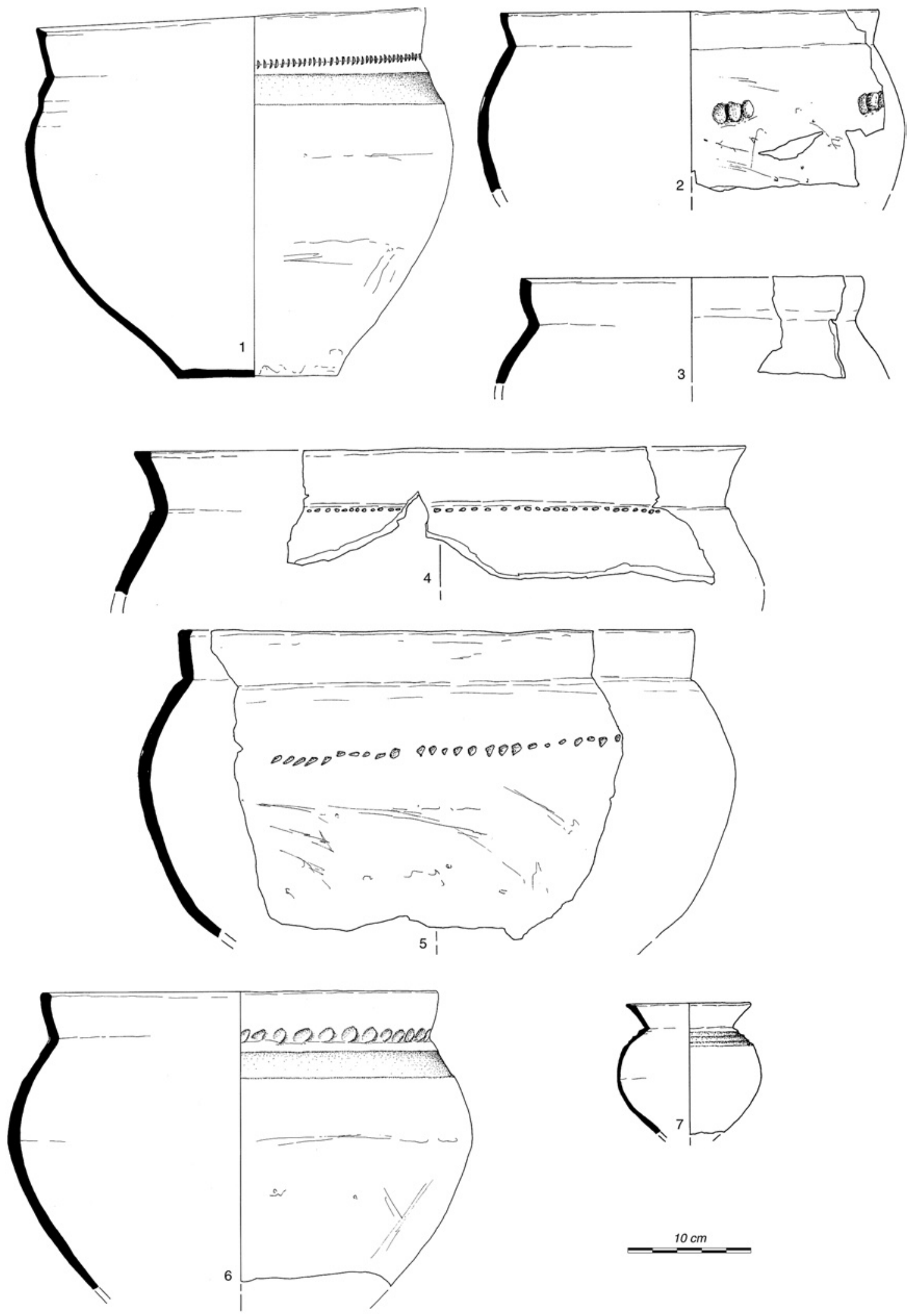

Fig. 11 - Mobilier céramique du fond de la fosse 25 du site de la Plaine. 
courant dès le Bronze final IIIa et pendant tout le Bronze final IIIb dans la moyenne vallée du Rhône et le Languedoc oriental : baume des Anges à Donzère, grotte des Cloches à Saint-Martin-d'Ardèche ou grotte 1 de Castelvielh (Vital, 1990, fig. 50, n ${ }^{\text {os }} 6$, 7 et 9; Vital, 1987, fig. 13, nos 1 à 5; Dedet et Pène, 1991, fig. 14 , fig. $15, \mathrm{n}^{\circ} 1$ et fig. $16, \mathrm{n}^{\text {os }} 1$ à 4 ). L'organisation de ces décors, où une ligne d'impressions souligne une ou plusieurs cannelures larges, est cependant différente de celle reconnue sur les céramiques de notre série.

\section{Forme exceptionnelle et objets divers en céramique}

Un petit "godet" de la fosse A10 (fig. 3, n 13), dont la panse est totalement recouverte de fines cannelures profondément marquées, ne trouve pas de comparaison directe. Nous noterons simplement qu'un traitement analogue est connu sur une jatte de l'oppidum de Rochefort à Varces-Allières-et-Risset dans l'Isère (Bocquet, 1969, fig. 104, n 23). L'absence de contexte sûr rend l'attribution de ce vase à une phase ancienne ou moyenne du Bronze final impossible.

Plusieurs fragments de croissants d'argile ou "chenets" à cornes courtes non décorés ont été trouvés dans la fosse SLP 25 (fig. 7, $\mathrm{n}^{\text {os }} 32$ et 33). Un tesson de la fosse A 2 pourrait également appartenir à une telle pièce (fig. 7, n ${ }^{\circ} 31$ ). La fonction précise de ces objets, connus en grande quantité dans les habitats et dans une moindre mesure dans les sépultures de la deuxième moitié du Bronze final, reste énigmatique. Néanmoins, la majorité des auteurs semble s'accorder pour leur donner une signification cultuelle, peut-être en relation avec une représentation symbolique de taureau (Rychner, 1988; Kossack, 1990). Un fragment de tore (ou anneau de suspension) provient de la fosse A14 (fig. 5, $\mathrm{n}^{\circ} 11$ ). Bien que de nombreux doutes subsistent quant à l'interprétation de ce type de mobilier, il reste tout à fait vraisemblable qu'ils aient pu servir à maintenir des vases à fond étroit ou pointu lors de leur séchage ou de leur cuisson (Bocquet et Couren, 1975). Signalons enfin la présence d'une fusaïole non décorée dans la fosse A11 (fig. 6, $\mathrm{n}^{\circ}$ 16).

\section{SYNTHÈSE}

Jusqu'à une date récente, les séries mobilières rhodaniennes attribuées au Bronze final IIIa restaient l'exception (Vital, 1990, p. 120). Alors que les phases précédente et suivante (Bronze final IIb et IIIb) s'avéraient mieux documentées, il demeurait difficile d'isoler le matériel rapportable à la seconde moitié du $\mathrm{XI}^{\mathrm{e}}$ siècle et au courant du $\mathrm{X}^{\mathrm{e}}$ siècle av. J.-C., dates communément admises pour cette période (Voruz, 1989). La conduite de nouveaux travaux permet à l'heure actuelle de pondérer cette carence :

- fouilles de la grotte du Gardon, du site de hauteur de Saint-Alban et de la ZAC des Perches (Voruz, 1991; Stahl, 1991; Treffort, 1993; Hénon, 1999);

- étude et réexamen de l'ensemble du mobilier issu des fouilles anciennes de la grotte des Cloches et de la Balme à Gontran (Vital, 1987; Treffort, Nicod et coll., 2000);

- nouvelles données concernant les sites lacustres corrigeant la supposition de l'absence d'occupation des rives des lacs alpins entre les derniers quarts des $\mathrm{XI}^{\mathrm{e}}$ et $\mathrm{X}^{\mathrm{e}}$ siècles av. J.-C. (Bocquet et al., 1988; Marguet, 2002). Il en est ainsi de la datation des sites de Duingt, le Roselet, de Veyrier-du-Lac, Vieugy-Nord et de Conjux, les Côtes dont le mobilier archéologique est difficilement exploitable, et surtout de la station de Tougues, ensemble 2 (Billaud et Marguet, 1992).

Il apparaît désormais possible de dégager quelques propositions pouvant aider à la définition des corpus céramiques de la fin du $\mathrm{XI}^{\mathrm{e}}$ et du $\mathrm{X}^{\mathrm{e}}$ siècle av. J.-C. pour un secteur géographique couvrant la vallée du Haut-Rhône français.

\section{Chronologie}

Sur le plan diachronique, en se référant en premier lieu aux séries du Bronze final $\mathrm{IIb}$ du nord de la région Rhône-Alpes (Pérouges-Croix Tombée, Vénissieux-le Vieux-Bourg, La Balme-grotte de la Balme), nous constatons, principalement dans la céramique fine, un certain nombre de différences dans la composition des corpus. La première et la plus marquante est l'absence ou quasi-absence des formes caractéristiques du début du RSFO (Rhin-Suisse-France orientale) dans les fosses des Estournelles et de la Plaine. C'est le cas des coupes à profil simple ou segmenté à décor de larges cannelures internes, d'arceaux peignés ou de motifs complexes incisés. La représentation des gobelets à épaulement larges ou étroits est également très faible. Les gobelets ovoïdes ainsi que les jattes arrondies semblent, par contre, constituer des nouveautés qui ne perdureront pas au-delà de la fin du $\mathrm{X}^{\mathrm{e}}$ siècle av. J.-C. Les coupes à rebord ne trouvent pas d'occurrences dans les séries régionales attribuées au Bronze final IIb. Leur représentation, de l'ordre de 3,7 \% à Simandres, est presque identique sur le site des Barlières à la fin du Bronze final IIIb (4,71 \%; Thiériot, 2000, fig. 59). La fragmentation des jarres à rebord ne permet pas encore de définir une évolution dans leur aspect général, bien qu'elles semblent le plus souvent dotées d'un col rectiligne développé se terminant par un petit marli durant le Bronze final IIb (Joly et Taras, 1994, pl. 26). Cependant, leur fréquence pourrait être significative : moins de $5 \%$ à Vénissieux ${ }^{1}$ contre près de $15 \%$ aux Estournelles et à la Plaine, chiffre de nouveau très proche de celui obtenu pour le mobilier des Barlières (15,63\%; Thiériot, 2000, fig. 59). La forme des pots à rebord ne semble pas présenter de variations importantes avec la phase antérieure. La représentation de certaines caractéristiques comme les cols cintrés ou la position des décors est, en effet, assez constante (la proportion de décors situés sur le point d'inflexion ou la partie supérieure des panses s'établit par exemple aux alentours de $65 \%$ à Venissieux pour $70 \%$ aux Estournelles et à la Plaine). Le pourcentage de lèvres ornées est en revanche largement moindre pour les 
séries de Simandres (10\% contre $46 \%$ sur le site du Vieux-Bourg). La confrontation avec la série des Barlières montre, par contre, des différences sensibles. Sur ce site, la fréquence des cols cintrés n'atteint pas $7 \%$ et celle des décors appliqués sur la partie supérieure de la panse ne dépasse pas $10 \%$ (Thiériot, 2000, tabl. 16 et fig. 49b).

En fonction de ceci et des comparaisons développées plus haut, nous proposons la définition de quatre groupes de caractères typologiques pour les corpus céramiques attribuables à la seconde moitié du $\mathrm{XI}^{\mathrm{e}}$ et au $\mathrm{X}^{\mathrm{e}}$ siècle av. J.-C. dans le nord de la région RhôneAlpes (fig. 12) :

- le premier d'entre eux est défini de manière "négative". Il est constitué de formes et caractéristiques, très répandues durant l'Âge du Bronze final IIb (gobelets à épaulement, coupes à profil segmenté, décors de larges cannelures, décors peignés ou incisés complexes...), absentes ou largement minoritaires dans l'ensemble des séries attribuées au Bronze final IIIa du secteur considéré ;

- le second groupe comprend les caractères, déjà répandus antérieurement, dont la représentation reste forte. Il semble concerner principalement certaines particularités des pots à rebord (rebords cintrés et position des décors);

- le groupe 3 correspond à un ensemble de formes considérées comme spécifiques. Nous les définissons comme des nouveautés qui, dans l'état actuel de la documentation, ne semblent connues ni dans le Bronze final IIb ni dans le Bronze final IIIb. Il s'agit
(1)

(2)

(3)

(4)
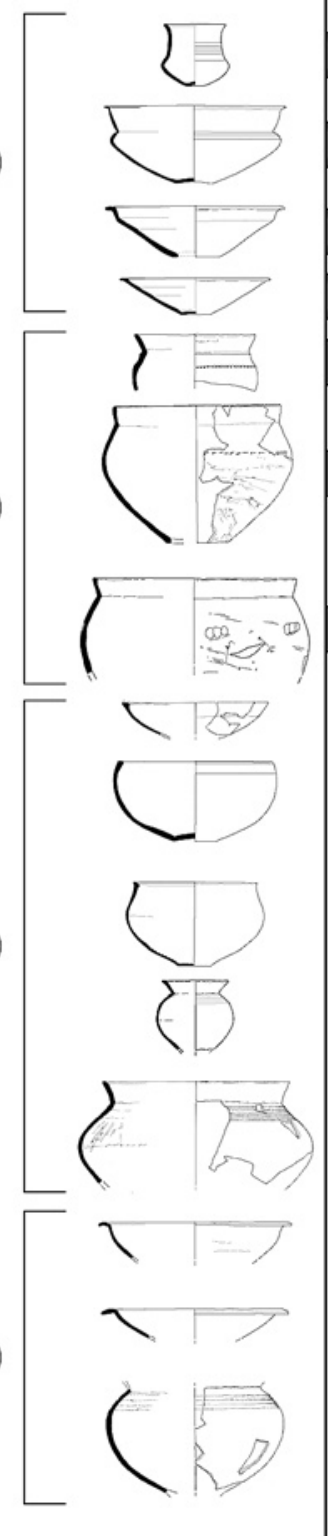

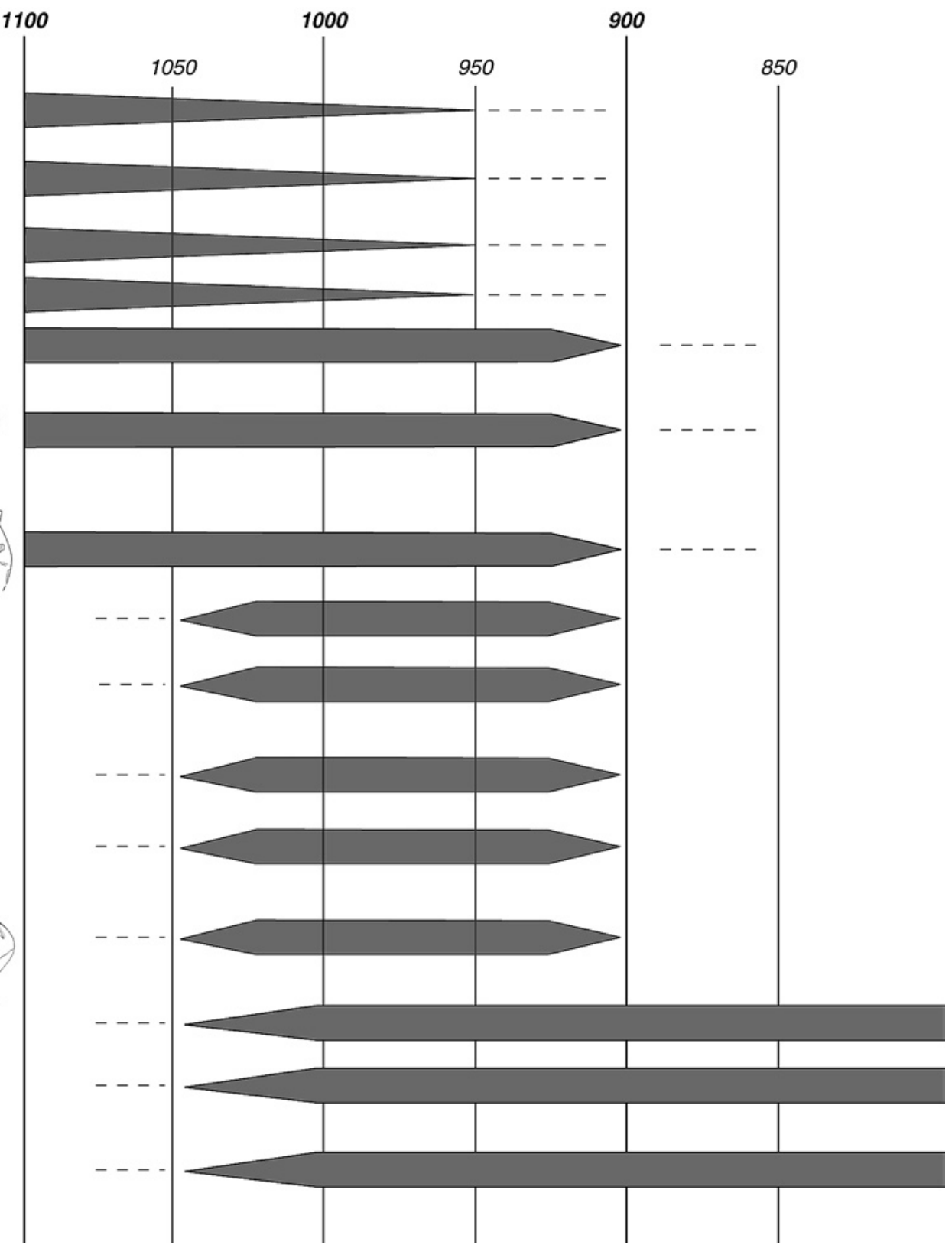

Fig. 12 - Proposition de groupes de caractères typologiques pouvant définir le mobilier céramique du $\mathrm{X}^{\mathrm{e}}$ siècle av. J.-C. pour le nord de la région Rhône-Alpes (les dates et rythmes d'apparition et de disparition sont donnés à titre indicatif). 
en premier lieu des jattes à profil arrondies et des gobelets ovoïdes. Nous proposons, avec réserve et dans la mesure où ces types ne paraissent pas représentés dans les séries du Bronze final IIIb local, de leur associer les coupes à profil concave et lèvres épaissies ainsi que les jarres à rebord à panse anguleuse (fig. 5, $\mathrm{n}^{\circ} 10$, avec un rebord décoré de deux cannelures internes et fig. $9, \mathrm{n}^{\circ} 33$ );

- le dernier groupe réunit les formes nouvelles qui perdureront pendant toute la durée du $\mathrm{IX}^{\mathrm{e}}$ siècle av. J.-C. (coupes à rebord et jarres à rebord à panse arrondie).

L'unique ambition de cette démarche de classement, obligatoirement simplificatrice, est d'offrir un premier cadre de réflexion. En effet, la multiplication d'analyses typologiques quantifiées sera seule à même de confirmer et surtout d'affiner ces propositions. Nous avons tenté, à titre d'exemple, d'appliquer une méthode de ce type sur les fosses des Estournelles et de la Plaine, bien que cette démarche paraisse audacieuse au regard de la représentativité statistique des séries. Nous avons postulé l'existence, sur la base des datations ${ }^{14} \mathrm{C}$, de deux ensembles chronologiques distincts au sein de nos structures :

- un ensemble récent est formé des fosses SLP 25 et A2. Les dates obtenues sur ces structures sont très proches et toutes deux issues du fond des creusements. Elles paraissent indiquer un bon terminus post-quem pour le début de leur fonctionnement qui s'est probablement déroulé sur plusieurs années (Blaizot et Thiériot, 2000, p. 235-237);

- la date, plus ancienne, réalisée sur des matériaux prélevés dans le comblement secondaire de la fosse A14, permet de supposer de sa relative antériorité vis-à-vis du groupe SLP 25/A2. Nous lui associons la fosse A10 (présence de tessons appartenant à un même vase) ainsi que, de manière plus arbitraire, les structures A11 et A12.

La comparaison des fréquences de chaque type céramique dans les corpus associés à ces deux groupes (fig. 13) montre une plus forte représentation des vases des groupes 3 et 4 (formes nouvelles) dans les fosses SLP 25 et A2, supposées récentes. Les gobelets à épaulement (groupe 1) sont par contre plus fréquents dans les structures les plus anciennes. Cette évolution pourrait constituer un début de vérification de nos hypothèses typochronologiques. La proximité des dates obtenues pour les deux ensembles conduit cependant à la plus grande prudence : ces différences peuvent être simplement dues au hasard (comme pourraient en témoigner les variations importantes dans les pourcentages de coupes et de pots à rebord). Il n'en demeure pas moins que ce type de démarche mérite d'être généralisé sur d'autres séries en mobilisant des critères descriptifs plus fins.

La question de l'évolution générale des corpus céramiques entre les $\mathrm{XIII}^{\mathrm{e}}$ et $\mathrm{IX}^{\mathrm{e}}$ siècles av. J.-C. (Bronze final IIb à IIIb) en termes de rupture ou continuité ne semble pas pouvoir être abordée de

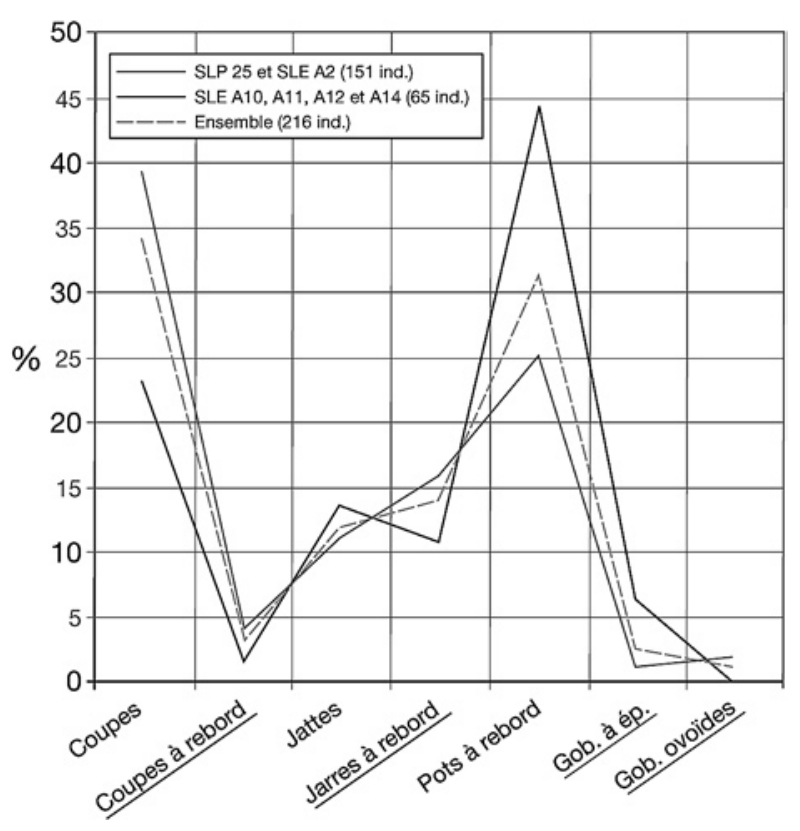

Fig. 13 - Tentative de comparaison proportionnelle entre les ensembles céramiques des fosses SLP 25/SLE A2 d'une part et SLE A10/A11/A12/ A14 d'autre part. Les fréquences des types soulignés pourraient avoir une valeur chronologique.

manière satisfaisante. Seuls deux aspects seront soulignés :

- l'absence ou la sous-représentation d'attributs typologiques largement répandus durant le Bronze final IIb et considérés comme caractéristiques du groupe RSFO sont attestées dans l'ensemble des séries attribuées au Bronze final IIIa (les Estournelles, la Plaine, ZAC des Perches, grotte du Gardon, station de Tougues). Elles semblent être sensibles dès le début du $\mathrm{X}^{\mathrm{e}}$ siècle av. J.-C.;

- une partie du cortège typologique des séries du IX siècle av. J.-C. semble, par contre, déjà présente dans le mobilier céramique de ces sites (groupe 4 : coupes à rebord et jarres à rebord). La mise en place des corpus propres à cette période ne semble pas s'effectuer avant l'extrême fin du $\mathrm{X}^{\mathrm{e}}$ siècle av J.-C.

L'interprétation de ces observations dépendra d'une part de la signification que l'on donnera à chacune d'entre elles et d'autre part de la rapidité de leur mise en œuvre. Nous pouvons envisager le déroulement d'un processus continu de disparition "d'archaïsmes" et d'apparition de nouveautés tendant vers l'équilibre et se déroulant sur une période relativement longue. Ce postulat, qui implique obligatoirement la présence de phases intermédiaires encore inconnues, traduirait une évolution sans rupture typologique majeure, à l'instar de ce qui est pressenti dans la région neuchâteloise (Rychner et coll., 1988). Nous pouvons par contre considérer une durée courte et donner une signification sémiologique forte aux formes et décors du groupe 1. La valeur heuristique de leur disparition sera alors beaucoup plus grande. Dans ce second cas, l'hypothèse de l'existence d'une rupture typologique au Bronze final IIb/IIIa pourra être posée. 
La documentation disponible ne permet pas de trancher entre ces deux propositions. Nous constatons que :

- le cadrage chronologique précis des séries du Bronze final IIb reste à faire;

- les sites attribués au Bronze final IIIa ayant fait l'objet de datations sont globalement plutôt situés dans le $\mathrm{X}^{\mathrm{e}}$ siècle av. J.-C. et peuvent être considérés comme relativement récents. Dans ce contexte, les phases intermédiaires envisagées dans le cadre d'une évolution continue pourraient trouver place durant le $\mathrm{XI}^{\mathrm{e}}$ siècle (existence d'un faciès "IIIa ancien"?);

- les distances typologiques séparant les diverses phases de la fin de l'Âge du Bronze ne sont pas exactement mesurées. Les propositions présentées ici ne peuvent que refléter partiellement une réalité plus complexe.

\section{Hypothèses géographiques}

La mise en perspective des quelques comparaisons interrégionales développées dans cet article avec divers travaux concernant le mobilier céramique de la période Bronze final IIb à IIIb médio-rhodanien nous conduit à proposer, avec prudence, quelques hypothèses géographiques. Celles-ci, principalement fondées sur des constats de présence ou d'absence de certaines caractéristiques typologiques, ne traduisent, en effet, qu'un moment de la recherche fortement contraint par l'état de la documentation disponible. Les limites ainsi définies n'ont qu'une valeur géographique indicative, leurs situations exactes devant être précisées à l'avenir (introduction de nouvelles références, reprise de collections anciennes...).

La synthèse la plus récente sur l'impact du groupe RSFO dans la moyenne vallée du Rhône a montré que les ensembles les plus purs (nécropole de la Croix Tombée à Pérouges) ne semblaient pas dépasser le nord de la région Rhône-Alpes (Vital, 1988). Vers le sud, les éléments typiquement RSFO sont encore présents, mais des mélanges tendent à s'effectuer engendrant la création de groupes mixtes (associations) ou de variantes locales (adaptations). Le renouvellement conséquent de la documentation concernant le Bronze final IIb, dans la région lyonnaise (ZAC des Perches à SaintPriest : Hénon, 1999; sites de Vénissieux : Joly et Taras, 1994...) ou la moyenne vallée du Rhône (site de Laprade à Lamotte-du-Rhône, Vaucluse; Billaud, 1999) ne devrait pas manquer d'apporter de nouveaux éléments de réponses. En direction des Alpes, la pénétration d'influences RSFO est, désormais, largement supposée (Ozanne et Vital, 1999, p. 529). Nous retiendrons, pour l'instant, l'hypothèse d'un impact nord oriental très sensible dans une grande partie nord de la région Rhône-Alpes au Bronze final IIb (fig. 14).

L'accroissement du nombre de sites régionaux attribués au Bronze final IIIa permet de proposer les remarques suivantes pour cette période :

- certaines formes connaissent une répartition très large. C'est le cas des jattes à profil arrondies connues

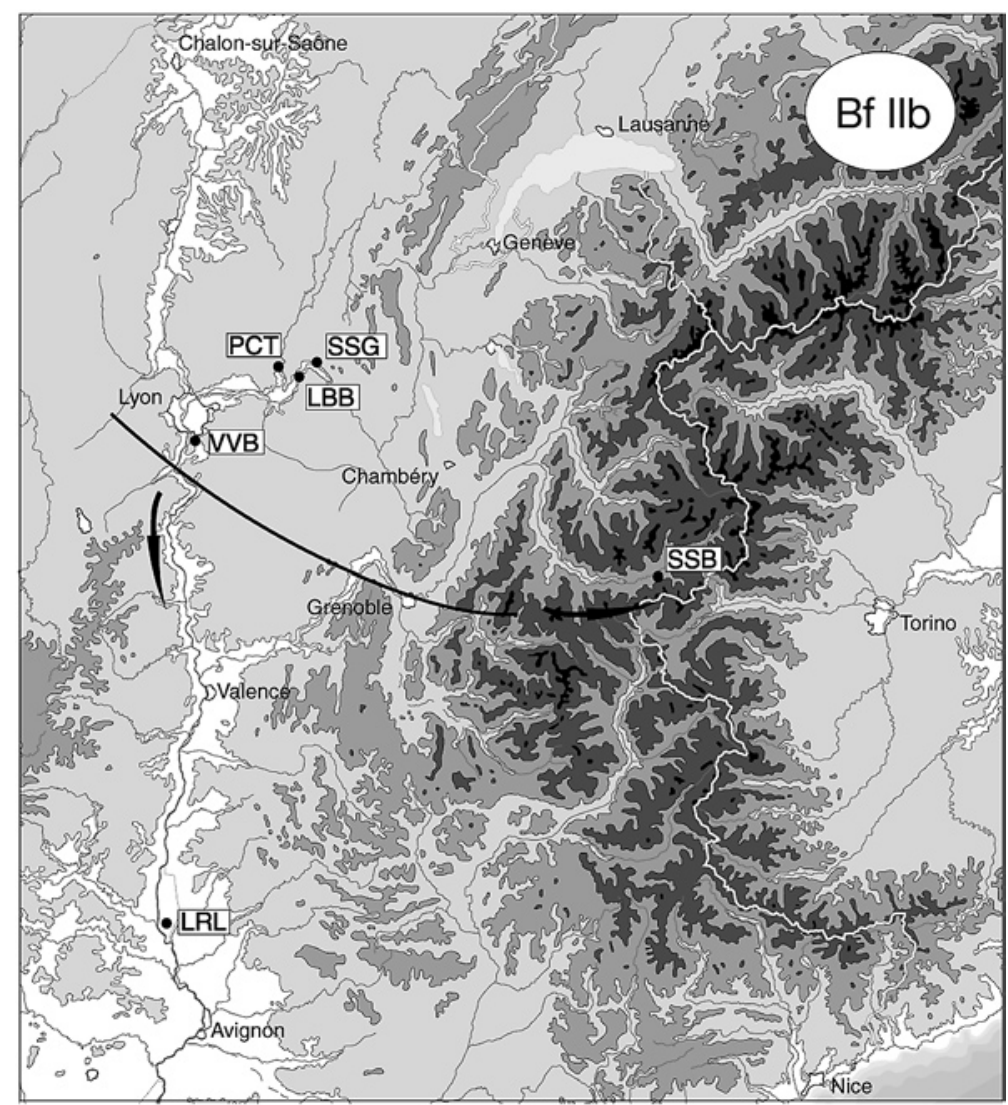

Fig. 14 - Localisation indicative d'une limite typologique reconnue pour l'Âge du Bronze final IIb (VVB : Vénissieux - le Vieux-Bourg; SSB : Sollières-Sardières - la Balme; LRL : Lamotte-duRhône - Laprade; SSG : Saint-Sorlin-enBugey - grotte des Gitans; PCT: Pérouges - la Croix Tombée; LLB : LaBalme-les-Grottes - grottes de la Balme). 
Fig. 15 - Localisation indicative d'une limite typologique reconnue pour l'Âge du Bronze final IIIa (SAC : Sainte-Anastasie - grotte 1 de Castelvielh; SPP : Saint-Priest - ZAC des Perches; SLE/ SLP : Simandres - les Estournelles et la Plaine; SMC : Saint-Martin-d'Ardèche - grotte des Cloches; CSG : Chalon-surSaône - le Gué-des-Piles; CLT : Chenssur-Léman - Tougues; ABG : Ambérieuen-Bugey - grotte du Gardon).

Fig. 16 - Localisation indicative des limites typologiques reconnues pour l'Âge du Bronze final IIIb (SFG : SaintFerréol-Trente-Pas - les Gandus; SBB : Serrières-de-Briord - les Barlières; MPC : Montagnieu - le Pré de la Cour; CC : Châtillon - Chindrieux; DBA : Donzère - baume des Anges).
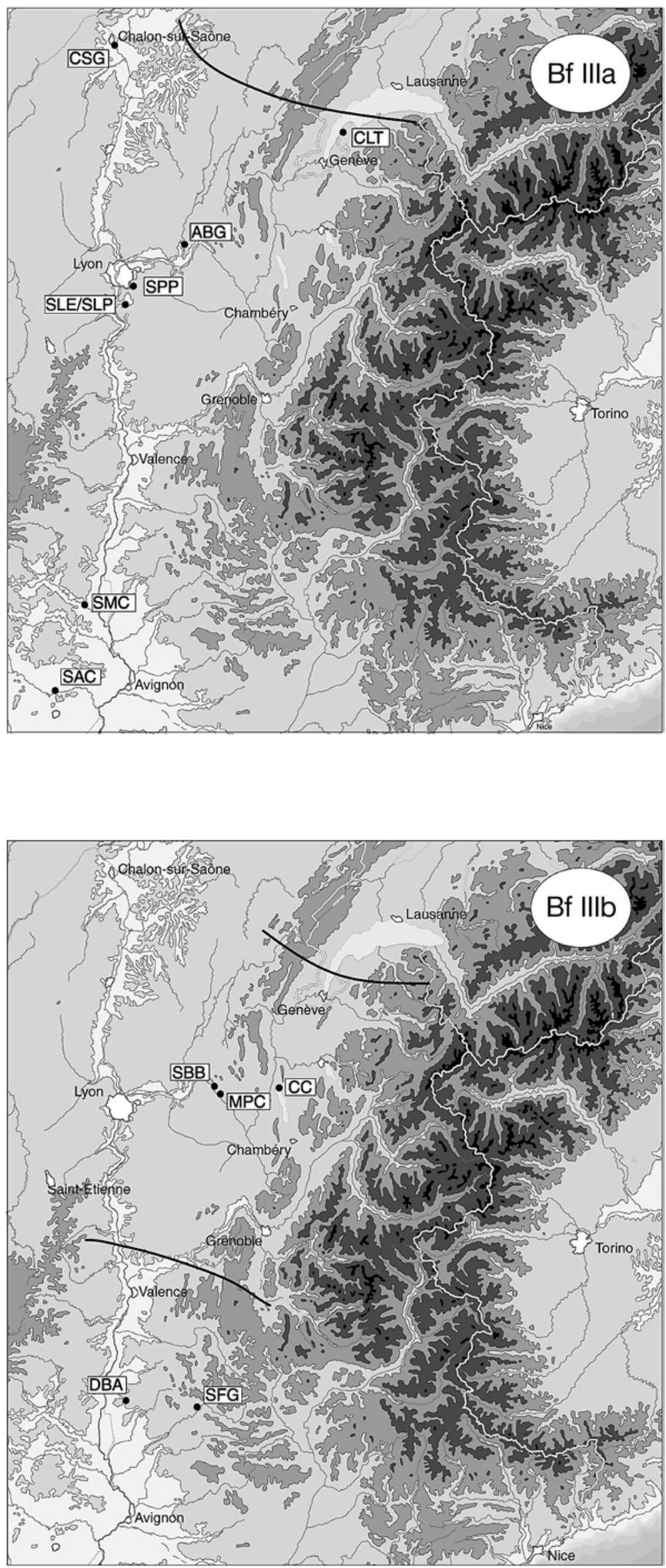
en Languedoc, moyenne vallée du Rhône, Jura, Val de Saône et Plateau suisse. Leurs dates d'apparition, connues ou supposées, semblent pratiquement synchrones sur l'ensemble de ce vaste secteur;

les comparaisons avec les sites suisses et franc-comtois traduisent des différences sensibles. Certains types ou décors, fréquents dans ces deux régions (gobelets à épaulement, décors peignés ou incisés complexes...), sont inconnus ou très rares en région Rhône-Alpes. A contrario, des formes bien représentées aux Estournelles et à la Plaine (coupes à rebord, jarres à rebord, gobelet ovoïde...) paraissent absentes ou minoritaires en domaine jurassien et en Suisse occidentale. Dans l'état actuel de la documentation, le seul ensemble présentant des affinités avec le Bronze final IIIa à "décor riche" du Plateau suisse ou du Jura est issu d'une incinération observée à Hières-sur-Amby dans l'Isère (inédit, renseignement R. Convert et J. Vital). L'hypothèse de l'existence d'une carence documentaire pouvant expliquer les disparités reconnues ne peut donc être totalement exclue. Cependant, l'augmentation sensible des corpus disponibles conduit à la considérer comme de plus en plus improbable. Notons à cet égard que le mobilier de Tougues, ensemble 2, dont les dates dendrochronologiques sont bien en phase avec celles de Hauterive zone D ou de Cortaillod-Est, se distingue sensiblement de celui connu sur ces sites;

- bien que la documentation disponible pour la vallée de la Saône soit encore limitée, les similitudes relevées avec le mobilier du site du Gué-des-Piles permettent de postuler d'une bonne proximité typologique avec le nord de la région Rhône-Alpes.

L'une des grandes nouveautés de cette période serait donc la mise en place d'une limite géographique, isolant, d'après la typologie céramique, la FrancheComté et le Plateau suisse d'une région comprenant le Jura méridional, la haute vallée du Rhône français et vraisemblablement la vallée de la Saône (fig. 15). Les connexions observées avec les séries méridionales (grotte des Cloches, grotte I de Castelviehl) doivent être précisées. Elles ne permettent pas d'associer avec certitude la moyenne vallée du Rhône français (Drôme, Ardèche, Gard...) au secteur nord rhodanien.

Deux "frontières typologiques" sont maintenant avérées durant le Bronze final IIIb. Vers le nord, d'une part, où des disparités sont observées, au moins avec les ensembles helvétiques, confirmant la continuité du phénomène sensible dès la période précédente (fig. 16). D'autre part, les comparaisons conduites entre le mobilier céramique du site des Barlières et celui du site des Gandus ont montré de grandes divergences attestant d'une certaine autonomie typologique entre le sud et le nord de la région Rhône-Alpes durant cette période (Thiériot, 2000).

\section{CONCLUSION}

En 1988, P. Brun définissait le Bronze final IIIa comme étant “... probablement plus un ensemble de traits culturels propres à la région suisse occidentale et Franche-Comté méridionale qu'une étape chronologique stricto sensu" (Brun, 1988, p. 601). Cette observation était alors fondée sur les difficultés d'identification d'un corpus céramique spécifique à cette phase dans des secteurs périphériques (Bassin parisien et vallée du Rhône par exemple : Brun, 1986; Blanchet et Talon, 1987; Vital, 1990). L'hypothèse de l'existence de carences documentaires pouvait, cependant, expliquer l'absence du faciès Bronze final IIIa caractéristique à "décors riches" dans ces régions. Elle alimentait également les polémiques autour de la datation du site du Gué-des-Piles qui semblait trop ancienne au regard du mobilier considéré comme rapportable à un Bronze final IIIb ancien (Lambert et al., 1988; Vital dir., 1993, p. 222).

L'examen du mobilier céramique des Estournelles et de la Plaine conduit à relativiser cette dernière proposition. Il permet, en effet, de caractériser, pour le nord de la région Rhône-Alpes, un assemblage typologique propre au $\mathrm{X}^{\mathrm{e}}$ siècle av. J.-C. Les proximités relevées avec le site châlonnais et plusieurs autres gisements rhodaniens de datations similaires semblent indiquer qu'il ne définirait pas un stade ancien du Bronze final IIIb mais une phase antérieure distincte de mieux en mieux documentée. En l'absence d'un cadrage chronologique précis des nombreuses séries du Bronze final IIb régional, il demeure difficile, par contre, d'appréhender les modalités de l'évolution des corpus céramiques entre les $\mathrm{XII}^{\mathrm{e}}$ et $\mathrm{IX}^{\mathrm{e}}$ siècles av J.-C. La réalisation de datations absolues et la pratique d'approches typologiques quantifiées seront des préliminaires essentiels pour aborder ce problème fondamental.

Une certaine prise d'autonomie vis-à-vis du complexe RSFO pourrait donc être sensible dans une région circum-jurassienne dès le $\mathrm{X}^{\mathrm{e}}$ siècle av. J.-C. Cette conjecture, à l'heure actuelle uniquement fondée sur des critères typologiques, devra être examinée à la lumière d'autres aspects sociaux et culturels (habitat, sépultures...). Faudrait-il y voir les prémices du morcellement culturel observé dès le Bronze final IIIb dans des régions plus septentrionales (Brun, 1986, p. 6667) ? À l'heure actuelle, l'origine et les motifs de mise en œuvre de cette dynamique restent inconnus (évolution sur place ou apport d'éléments exogènes). Dans une telle perspective et malgré leur isolement, l'originalité des gestes funéraires reconnus aux Estournelles et à la Plaine pourrait être significative.

\section{NOTE}

(1) Les pourcentages proposés pour la céramique du site de Vénissieuxle Vieux-Bourg sont calculés d'après le mobilier présenté dans le rapport de fouille (Joly et Taras, 1994, pl. 1 à 28). 


\section{RÉFÉRENCES BIBLIOGRAPHIQUES}

BERNATZKY-GOETZE M. (1987) - Mörigen. Die spätbronzezeitlichen Funde, Antiqua 16, publication de la Société suisse de Préhistoire et d'Archéologie, Bâle, 136 p., 176 pl.

BILLAUD Y. (1999) - Laprade, Lamotte-du-Rhône (Vaucluse) : un habitat de plaine à architecture de terre au Bronze final IIb, Bulletin de la Société préhistorique française, t. 96, n 4, p. 607-621.

BILLAUD Y., MARGUET A. (1992) - Le site Bronze final de Tougues à Chens-sur-Léman (Haute-Savoie). Stratigraphie, datations absolues et typologie, Actes du $116^{e}$ congrès des Sociétés savantes, Chambéry, 1991, commission de Pré- et Protohistoire, p. 311-347.

BILLAUD Y., MARGUET A., SIMONIN O. (1992) - Chindrieux, Châtillon (lac du Bourget, Savoie). Ultime occupation des lacs alpins français à l'Âge du Bronze?, Actes du $116^{e}$ congrès des Sociétés savantes, Chambéry, 1991, commission de Pré-et Protohistoire p. $277-310$

BLAIZOT F., THIÉRIOT F. et coll. (2000) - Un rituel funéraire original de la fin de l'Âge du Bronze. Les sites des Estournelles et de la Plaine à Simandres (Rhône), Gallia Préhistoire, 42, p. 195-256.

BLANCHET J.-C., TALON M. (1987) - L'éperon barré du "Camp César" à Catenoy (Oise), à l'Âge du Bronze final. Premiers résultats, Actes du $22^{e}$ Congrès préhistorique de France, Les relations entre le continent et les îles Britanniques, Lille, 1988, Revue archéologique de Picardie, Société préhistorique française, p. 189-210.

BOCQUET A. (1969) - L'Isère préhistorique et protohistorique, Gallia Préhistoire, XII, 1, p. 121-258, 2, p. 273-400.

BOCQUET A., COUREN J.-P. (1975) - Le four de potier de Sévrier, Haute-Savoie (Âge du Bronze final), Études préhistoriques, 9.

BOCQUET A., MARGUET A., ORCEL C. et A. (1988) - Datations absolues sur les stations littorales de l'Âge du Bronze final dans les Alpes du Nord, in P. Brun et C. Mordant dir., Le groupe Rhin-SuisseFrance orientale et la notion de civilisation des Champs d'Urnes, Actes du colloque international de Nemours, 1986, Mémoires du musée de Préhistoire d'Île-de-France, 1, p. 435-444.

BOCQUET A., REYMOND J. (1976) - La grotte de la Balme (Isère). Néolithique et âges des Métaux dans les Alpes françaises, IX ${ }^{e}$ congrès de l'UISPP, livret-guide de l'excursion A9, Nice, p. 192-199.

BONNAMOUR L. (1989) - L'habitat Bronze final du Gué-des-Piles à Chalon-sur-Saône (Saône-et-Loire). Étude archéologique, Gallia Préhistoire, 31, p. 159-189.

BONNAMOUR L., MARINVAL P., MARGUERIE D., LAMBERT G., LAVIER C., POULAIN T. (1990) - Les habitats du Bronze final III dans la moyenne vallée de la Saône, au sud de Chalon et le Gué-desPiles, Un monde villageois. Habitat et milieu naturel en Europe de 2000 à 500 avant J.-C., Lons-le-Saunier, p. 97-111.

BORRELLO M.-A. (1986) - Cortaillod-Est, un village du Bronze final, 2. La céramique, Archéologie neuchâteloise, 2, éd. du Ruau, SaintBlaise, 100 p., 41 fig., 78 pl.

BORRELLO M.-A. (1992) - Hauterive-Champréveyres, 6. La céramique du Bronze final, zones $D$ et $E$, Archéologie neuchâteloise, 14, éd. du Ruau, Saint-Blaise, 88 p., 64 fig., 72 pl. hors-texte.

BORRELLO M.-A. (1993) - Hauterive-Champréveyres, 7. La céramique du Bronze final, zones A et B, Archéologie neuchâteloise, 15, éd. du Ruau, Saint-Blaise, 91 p., 49 fig., 138 pl. hors-texte.

BRUN P. (1986) - La civilisation des Champs d'Urnes : étude critique dans le Bassin parisien, Documents d'Archéologie française, 4, éd. de la Maison des Sciences de l'Homme, Paris, 172 p., 45 fig., 78 pl.

BRUN P. (1988) - L'entité Rhin-Suisse-France orientale; nature et évolution, in P. Brun et C. Mordant dir., Le groupe Rhin-Suisse-France orientale et la notion de civilisation des Champs d'Urnes, Actes du colloque international de Nemours, 1986, Mémoires du musée de Préhistoire d'île-de-France, 1, p. 599-620.

DAUGAS J.-P., VITAL J. (1988) - Éléments du groupe RSFO dans le Massif central français (Auvergne et Forez), in P. Brun et C. Mordant dir., Le groupe Rhin-Suisse-France orientale et la notion de civilisation des Champs d'Urnes, Actes du colloque international de Nemours, 1986, Mémoires du musée de Préhistoire d'Île-de-France, 1, p. 425434.

DAUMAS J.-C., LAUDET R. (1985) - L'habitat du Bronze final des Gandus à Saint-Ferréol-Trente-Pas, Études préhistoriques, 1 (198182), p. 1-32

DEDET B., CHARMASSON J. (1989) - L'habitat du Bronze final IIIb de Lombren à Vénéjan (Gard), Gallia Préhistoire, 31, p. 191-207.

DEDET B., MICHELOZZI A., PY M. (1978) - Ugernum, Protohistoire de Beaucaire, Cahier $n^{\circ} 6$ de l'Association pour la Recherche archéologique en Languedoc oriental, Caveirac, $156 \mathrm{p}$.

DEDET B., PÈNE J.-M. (1991) - La grotte I de Castelviehl à SainteAnastasie (Gard), Documents d'Archéologie méridionale, 14, p. 89223.

DUMONT A., TREFFORT J.-M. (1991) - Histoire et bilan des fouilles anciennes, Archéologie de la grotte du Gardon (Ain), Rapport de fouilles 1985-1990, Document du département d'Anthropologie et d'Écologie de l'université de Genève, 17, p. 43-63.

GROSS E. (1986) - Vinelz-Ländti. Grabung 1979. Die neolitischen und spätbronzezeitlichen Ufersiedlungen, Staatlicher Lehrmittelverlag, Bern, 185 p., 73 fig.

HÉNON P. (1999) - Occupation d'un espace au Bronze final IIb/IIIa les locus 1 et 2 de la "ZAC des Perches" à Saint-Priest (Rhône), mémoire de DEA, université de Franche-Comté, Besançon, 2 vol., 92 p., 75 fig.

JOLY J.-L., TARAS M. (1994) - Opération de la ZAC du Vieux-Bourg à Vénissieux (Rhône). Place de la Paix, Rapport de fouilles 1993, service régional de l'Archéologie de la région Rhône-Alpes.

KAENEL G., KLAUSENER M. (1990) - Quelques tombes à incinération du Bronze final ( $\mathrm{X}^{\mathrm{e}}$ siècle av. J.-C.) à Vidy (Lausanne, VD), Annuaire de la Société suisse de Préhistoire et d'Archéologie, 73, p. 51-82.

KOSSACK G. (1990) - Kultgerät, Weihegabe und Amulett aus spätbronzezeitlichen Seeufersiedlungen, Archéologie suisse, 13, 2, p. 89100.

LAMBERT G., LAVIER C., BONNAMOUR L. (1988) - Étude dendrochronologique préliminaire du Gué-des-Piles à Chalon-sur-Saône, in P. Brun et C. Mordant dir., Le groupe Rhin-Suisse-France orientale et la notion de civilisation des Champs d'Urnes, Actes du colloque international de Nemours, 1986, Mémoires du musée de Préhistoire d'Île-de-France, 1, p. 373-377.

MARGUET A. (2002) - Savoie. Lac du Bourget, Bilan scientifique du département des Recherches archéologiques subaquatiques et sousmarines, 2000, direction de l'Architecture et du Patrimoine, sousdirection de l'Archéologie, p. 117-137.

OZANNE J.-C., VITAL J. (1999) - Documents pour servir à l'identification des relations transalpines occidentales dans la deuxième moitié de 1'Âge du Bronze, in A. Beeching dir., Circulations et identités culturelles alpines à la fin de la Préhistoire. Matériaux pour une étude, Programme CIRCALP 1997-1998, Travaux du Centre d'Archéologie préhistorique de Valence, $\mathrm{n}^{\circ} 2$, p. 519-553.

PÉTREQUIN P., CHAIX L., PÉTREQUIN A.-M., PININGRE J.-F. (1985) - La grotte des Planches-près-Arbois (Jura). Proto-Cortaillod et Âge du Bronze final, Maison des Sciences de l'Homme, Paris, $273 \mathrm{p}$

RYCHNER V. (1979) - L'Âge du Bronze final à Auvernier (lac de Neuchâtel, Suisse) : typologie et chronologie des anciennes collections conservées en Suisse. Auvernier 1 et 2, Bibliothèque historique vaudoise, Cahiers d'Archéologie romande, 15 et 16, 116 et 324 p.

RYCHNER V. (1988) - Sépultures, lieux de culte et croyances à l'Âge du Bronze, Sépultures, lieux de culte et croyances, $5^{e}$ cours d'initiation à la Préhistoire et à l'archéologie de la Suisse, résumé des cours, Société suisse de Préhistoire et d'Archéologie, Bâle, p. 7391. 
RYCHNER V. et coll. (1988) - Dendrochronologie du groupe RhinSuisse dans la région neuchâteloise, in P. Brun et C. Mordant dir., Le groupe Rhin-Suisse-France orientale et la notion de civilisation des Champs d'Urnes, Actes du colloque international de Nemours, 1986 Mémoires du musée de Préhistoire d'île-de-France, 1, p. 125-136.

SENÉE A. (1988) - La nécropole à incinération des Basses-Godernes de Champagne-sur-Seine (Seine-et-Marne), in P. Brun et C. Mordant dir., Le groupe Rhin-Suisse-France orientale et la notion de civilisation des Champs d'Urnes, Actes du colloque international de Nemours, 1986, Mémoires du musée de Préhistoire d'Île-de-France, 1, p. $305-$ 316.

STAHL L.-I. (1991) - Saint-Alban locus A. Étude d'un site d'habitat du Bronze final IIIb, travail de diplôme dactylographié, département d'Anthropologie et d'Écologie, université de Genève, 118 p., 36 fig., $23 \mathrm{pl}$.

STUIVER M., REIMER P.J. (1993) - Extended ${ }^{14} \mathrm{C}$ database and revised CALIB radiocarbon calibration program, Radiocarbon, 35, p. 215230.

STUIVER M., REIMER P.J., BARD É., BECK J.W., BURR G.S., HUGHEN K.A, KROMER B., McCORMAC F.G., PLITCH J., SPURK M. (1998) - INTCALC98 Radiocarbon age calibration 24000 - 0 cal BP, Radiocarbon, 40, p. 1041-1083

THIÉRIOT F. (2000) - Le mobilier céramique de l'Âge du Bronze final IIIb du site des Barlières à Serrières-de-Briord (Ain). Tentative de caractérisation d'une production du IX $X^{e}$ siècle avant J.-C. dans la haute vallée du Rhône français, mémoire de maîtrise, université de Bourgogne, 2 vol., 105 p., 80 fig., 57 pl., annexes.

THIÉRIOT F., SAINTOT S. et coll. (1999) - La fosse néolithique du site des Estournelles à Simandres (Rhône), in A. Beeching dir., Circulations et identités culturelles alpines à la fin de la Préhistoire. Matériaux pour une étude, Programme CIRCALP 1997-1998, Travaux du Centre d'Archéologie préhistorique de Valence, $n^{\circ}$ 2, p. 403-425.

TREFFORT J.-M. (1993) - Saint-Alban-locus B. Du Bronze final IIIa au Hallstatt ancien. Stratigraphie, structures d'habitat et chronotypologie du sondage SA5 de l'éperon de Saint-Alban, commune de Creys-et-Pusignieu, Isère, mémoire de maîtrise dactylographié, université Lumière-Lyon II, 2 vol., 117 p., 113 fig.

TREFFORT J.-M., NICOD P.-Y et coll. (2000) - La Balme à Gontran à Chaley (Ain) : du Néolithique moyen au haut Moyen Âge dans une cavité du Jura méridional, Revue archéologique de l'Est, 50 (1999. 2000), p. 53-118
VITAL J. (1987) - La grotte des Cloches à Saint-Martin-d'Ardèche, Bulletin de la Société préhistorique française, t. 83, Études et Travaux, $11-12$, p. $503-545$

VITAL J. (1988) - Le groupe RSFO dans la moyenne vallée du Rhône, in P. Brun et C. Mordant dir., Le groupe Rhin-Suisse-France orientale et la notion de civilisation des Champs d'Urnes, Actes du colloque international de Nemours, 1986, Mémoires du musée de Préhistoire d'Île-de-France, 1, p. 445-457.

VITAL J. (1990) - Protohistoire du défilé de Donzère : l'Âge du Bronze dans la baume des Anges (Drôme), Documents d'Archéologie française, 28, éd. de la Maison des sciences de l'Homme, Paris, 145 p., 57 fig.

VITAL J. dir. (1993) - Habitats et sociétés du Bronze final au premier $\hat{A} g e$ du Fer dans le Jura. Les occupations protohistoriques et néolithiques du Pré de la Cour à Montagnieu (Ain), Monographie du CRA, 11, éd. CNRS, 253 p., 107 fig.

VORUZ J.-L. (1986) - Un habitat-refuge à la grotte des Gitans à SaintSorlin-en-Bugey (Ain), Spéléo 01, 10, p. 28-43.

VORUZ J.-L. (1989) - De l'utilité des datages radiocarbone pour l'ÂAe du Bronze à propos des stratigraphies de Saint-Alban et du Gardon, Éléments de Protohistoire en Rhône-Alpes, 1, Actes des rencontres Protohistoire de Rhône-Alpes, Lyon, 1988, p. 19-28.

VORUZ J.-L. (1991) - L'occupation de l'Âge du Bronze final IIIa, Archéologie de la grotte du Gardon (Ain). Rapport de fouilles 19851990, Document du département d'Anthropologie et d'Écologie de l'université de Genève, 17, p. 223-235.

VORUZ J.-L. dir. (1993) - La grotte du Gardon à Ambérieu-en-Bugey (Ain). Rapport de fouilles 1993, département d'Anthropologie, université de Genève, Société préhistorique rhodanienne, Ambérieu-enBugey, 270 p., 139 fig.

Franck THIÉRIOT

INRAP Grand-Est - Nord 12, rue de Méric, C.S. 80005, 57063 METZ Cedex 02 